\title{
Stability Analysis of Digital Microwave Power Amplifiers
}

\author{
Almudena Suarez, Fellow, IEEE, Andreas Wentzel, Member, IEEE, Wolfgang Heinrich, Fellow, IEEE
}

\begin{abstract}
This work presents a stability analysis of microwave power amplifiers (PA) driven by binary pulse trains, as in the case of class-S PAs. First, using a simplified digital PA test-bench in class-D configuration, different qualitative behaviors are obtained when varying the pulse width, including subharmonic and incommensurable oscillations. The mechanisms affecting the stability properties are studied with a harmonicbalance based formulation, by means of pole-zero identification and bifurcation detection. A sufficiently high number of harmonic components must be considered, together with Krylov decomposition for an efficient computation of the inverse of the Jacobian matrix. It is demonstrated that, when varying the pulse width, distinct pairs of complex-conjugate poles may shift to the right-hand side of the complex plane and, therefore, lead to different kinds of unstable behavior. This phenomenon is related to the dependence of the critical poles on the average value of the input signal. Boundaries of the various types of unstable behavior are traced in the plane defined by the pulse repetition rate and pulse width, using bifurcation detection techniques. All the predicted phenomena have been confirmed experimentally. In a second step, the algorithms derived from the simple class-D circuit are transferred to study the stability of a more complex tri-band class-S amplifier. It has been analyzed versus the input bitrate, obtaining a fully stable behavior that has been validated experimentally.
\end{abstract}

Index Terms - Digital power amplifier, class-D, class-S, stability analysis, bifurcation analysis.

\section{INTRODUCTION}

$\mathrm{T}$ he growing demands towards wideband, multi-band and multi-standard features go along with the need for highly flexible, highly efficient and cost-effective nextgeneration wireless communication architectures [1]. These requirements have been fueling research on the digital microwave power amplifier (DPA) during the last years [2][5], as the RF PA is the last analog part in the almost digitized base station. Well known in the audio region, the digital PA concepts like class $\mathrm{S}$ have been transferred more and more to

Manuscript received May 3, 2016. This work has been supported by the Spanish Government under contract TEC2014-60283-C3-1-R, the European Regional Development Fund (ERDF/FEDER) and the Parliament of Cantabria (12.JP02.64069).

A. Suarez is with the Communications Engineering Department, University of Cantabria, 39005, Santander, Spain (e-mail: almudena.suarez@unican.es).

A. Wentzel and W. Heinrich are with the Ferdinand-Braun-Institut, 12489 , Berlin, Germany (e-mail: Andreas.Wentzel@FBHBerlin.de;Wolfgang.Heinrich@FBH-Berlin.de). the microwave frequency range, with the final objective of a fully digital transmitter architecture. Several such PAs have been published [2]-[5], including multi-band [6] and H-bridge [5] versions. The final stage of these amplifiers is realized in class-D topology. Thus, class-S and class-D are closely related. Circuit-wise class D forms the basis of any class-S solution and in the literature sometimes both labels are used for the same architecture. All the work so far targets the digital transmitter architecture in mobile communications, most of it the infrastructure part, which is the focus of this paper as well. But one has to state that, in the microwave range, the digital PA approach is still very premature.

Regarding electrical stability, numerous mentions to the corresponding problems in class-D amplifiers can be found in scientific papers, application notes, etc [7]-[10]. For instance, the work [10] presents a detailed stability analysis of a class-D audio amplifier, which is modeled in Simulink, with ideal components and numerical integration blocks. However, the authors have not found any investigations of instability phenomena in such circuits described with detailed device models in the microwave range. This is the focus of this work. We present for the first time a complete stability analysis of a digital microwave power amplifier, which is important for further research on digital transmitter architectures in future wireless communication networks.

The first objective is to demonstrate that the stability of class-D and class-S amplifiers can be analyzed out with techniques based on harmonic balance (HB), such as polezero identification [11]-[12] and frequency-domain bifurcation detection [13]-[17] (note that it is not always possible to analyze complex circuit architectures in time domain). The second objective is the thorough investigation of the specificity of the instability problems in differential topologies, with emphasis on the way how the pulse scheme and circuit symmetries affect the stability properties.

First, a very simplified proof-of-concept digital PA circuit of class-D type is studied and realized, in order to analyze the influence of varying input pulses on the stability. This very basic circuit for a single frequency band helps understand the typical instability phenomena in digital PA circuits. As mentioned above the class-D topology is used for the final stage in digital amplifiers and, accordingly, it shows the basic behavior of the targeted more complex class-S circuits. The relative simplicity of the class-D circuit, on the other hand, enables an accurate and reliable comparison with time-domain integration, which was not feasible with the class-S amplifier. Afterwards, one can transfer the algorithms derived from this simple case to more complex class-S scenarios. Still there can 
be instabilities which occur with a more complex modulation scheme (and modulated signals) only, but this can be studied at a later stage. Given the present early state of development of microwave class-S PAs, it appears to be advisable to focus on the basic effects. On the other hand, the consideration of two different amplifiers, instead of only one, demonstrates the generality of application of the analysis methods.

As stated, the analysis has been carried out with techniques based on HB [11]-[17]. A sufficiently high number of harmonic terms must be considered, using Krylov decomposition [18]-[19] for an efficient computation of the inverse of the HB Jacobian matrix. The advantage of this frequency-domain analysis is its efficient combination with continuation methods to follow the evolution of the stability properties versus relevant parameters, such as the pulse amplitude, width and repetition rate. When having full access to the HB system and its Jacobian matrix, the most widely applied continuation methods are prediction-correction with parameter switching [20]-[21] and arc-length [22]-[26]. When using commercial software, the user can perform this continuation externally to the HB system, by applying parameter switching to an auxiliary generator (AG) [17], [27][28].

The stability analysis will rely on a small perturbation of the pulse-driven circuit about the periodic steady-state regime [11]-[17]. With respect to this perturbation, the circuit acts as a linear time-variant system [29]-[30]. This time variation is strongly influenced by the input-signal characteristics (shape, amplitude and frequency), which affect the steady-state solution and, hence, the nonlinear-element Jacobian matrix, playing a major role in the characteristic system [13]-[17], [30]. As will be shown, when varying the duty cycle, distinct real or complex-conjugate poles may become dominant, which is due to the biasing effect of the input pulse.

For some particular parameter values, the dominant pole(s) may cross the imaginary axis, giving rise to a bifurcation [29][30], or qualitative change of the solution stability properties. Bifurcation loci [14],[17] in terms of parameters such as the pulse width, pulse amplitude and duty cycle will be presented here for the first time to our knowledge. A time map traced in terms of the repetition rate and pulse width can help predict the overall behavior in modulated conditions. In fact, when reducing the modulation frequency, the stability properties tend to those obtained in the absence of a modulation [31]. On the other hand, when increasing the modulation frequency, there is usually a stabilization effect, as has been validated here with the envelope-transient method [32].

The paper is organized as follows. In Section II, the influence of the pulse train on the stability properties will be investigated with time- and frequency-domain techniques. Sections III and IV present the bifurcation analysis versus different pulse characteristics, together with a detailed experimental validation. In Section V, the stability of a triband class-D/class-S amplifier is analyzed versus the bitrate. Section VI concludes the paper.

\section{INFLUENCE OF THE PULSE TRAIN ON THE STABILITY PROPERTIES}

\section{A. Large-signal instability mechanisms}

For an intuitive understanding of the influence of an input signal on the stability properties, two simple representative cases will be considered: a single resonant network with negative-resistance expansion and a nonlinear parametric resonator, which correspond to the two main instability mechanisms in large-signal operation [11]-[17],[29]-[30]. The circuit in Fig. 1(a) will be initially considered. It contains a linear resonator $G-L-C$, a nonlinear voltage-controlled current source $i(v)$ (i.e., a nonlinear conductor) and a time-varying independent current source $i_{p}(t)$. The circuit is governed by the following nonlinear differential equation:

$$
\ddot{v}(t)+\frac{1}{C}\left(G+\frac{d i(v)}{d v}\right) \dot{v}(t)+\omega_{o}^{2} v-\frac{1}{C} \frac{d i_{p}(t)}{d t}=0
$$

where $\omega_{o}=1 / \sqrt{L C}$ is the linear-network resonance frequency. Provided that the damping term [the one affecting $\dot{v}(t)]$ fulfils $G+d i(v=0) / d v=G_{T}>0$, the circuit will be stable in small-signal conditions. However, in the presence of a large-signal periodic input source $i_{p}(t)$, at the frequency $\omega_{p}=2 \pi f_{p}$, an oscillation may arise if the damping term takes a negative value $G+d i / d v<0$ within certain time intervals, since this corresponds to an energy transfer from the nonlinear element $i(v)$ to the resonator. In average, there must be an excess negative conductance at the resonance frequency, influenced by the input source $i_{p}(t)$. In the case of a sinusoidal excitation, and using a simplified analysis based on the incremental describing function $G_{N}(V)$ of $i(v)$ [33] (of conductance type), this would correspond to $G_{N}(V)+G<0$ at the complex perturbation frequency $s$, where $V$ is the fundamental voltage amplitude at $\omega_{p}$. However, for a rigorous stability analysis one should take into account all the relevant harmonic terms. Due to the independency of $\omega_{p}$ and $s$, the poles will have the form $j k \omega_{p}+s$ [11]-[17],[34]-[35] where $k$ is an integer. For a small-signal $i_{p}(t)$, the poles will be $\sigma_{o} \pm j \omega_{o}+j k \omega_{p}$, where $\sigma_{o}=-G_{T} / 2 C<0$. However, when increasing the amplitude of $i_{p}(t)$ both the real and imaginary parts of $s$ will deviate continuously from their original values, crossing to the right-hand-side of the complex plane (RHS) in some cases.

The second mechanism is the parametric oscillation [27], [36]-[37]. A resonator containing an inductor $L$, resistor $R$ and nonlinear capacitor $c(v)$ will be considered. Under a smallsignal excitation $i_{p}(t)$, the resonance frequency is $\omega_{o}=1 / \sqrt{L C_{a v e}}$, where $C_{a v e}$ is the average capacitor value. Under a large-signal periodic excitation from $i_{p}(t)$ at $\omega_{p}$, an instability may arise due to the energy transferred from $i_{p}(t)$ to the resonator. Indeed, linearizing the capacitor current $i(t)=c(v) d v / d t$ about the periodic regime at $\omega_{p}$, in the presence of a small voltage perturbation, one may obtain negative resistance in some frequency intervals [17]. When 
the circuit is driven at a frequency $\omega_{p}$ near $\omega_{o}$, the input source tends to shift the original resonator poles towards $\sigma_{p} \pm j k \omega_{p}$, where the subindex in $\sigma_{p}$ stresses the dependence on $i_{p}(t)$. When it is driven near $2 \omega_{o}$ it tends to shift the poles towards $\sigma_{p} \pm j k \omega_{p} / 2$. In the two former cases, the crossing of the poles to the RHS will give rise to hysteresis and subharmonic oscillations, respectively. In more complex topologies, quasi-periodic regimes may also be observed [37].

In most previous works, the analysis of the impact of the input source has been limited to sinusoidal sources. Here the objective will be to study the impact of pulse excitation, under different values of the pulse width or duty cycle.

\section{B. Proof-of-concept circuit}

For the stability investigation, the proof-of-concept circuit shown in Fig. 1(b-c) will be considered. The circuit contains two PHEMT GaAs transistors ATF50189 in the low cost surface mount SOT-89 package. The two transistors, biased at the drain voltage $V_{\text {Drain }}=4 \mathrm{~V}$, are excited in odd-mode, as in a class-D configuration. The circuit is expected to operate at $f_{p}=800 \mathrm{MHz}$. Large signal instability can be achieved by simply tuning the values of the output network, without any additional feedback. Note that the purpose of this test circuit is to investigate the impact of the rectangular pulse drive on the stability properties of the differential topology, which is analogous to that used in the practical class-S amplifier presented in Section V. The advantage of the simple circuit in Fig. 1(b-c) is the possibility to validate the frequency-domain results with time-domain integration, which is not applicable to the circuit in Section $\mathrm{V}$.

The circuit in Fig. 1(b-c) is stable in the absence of an input signal. However, when driven with a rectangular pulse it exhibits a variety of instability phenomena, as verified with pole-zero identification [11]-[12], [35], transient simulations and measurements. In the following analyses, a periodic pulse train centered around zero volts, with total amplitude $V_{i n}$, (pulse excursion from $-V_{\text {in }} / 2$ to $V_{\text {in }} / 2$ ) is introduced. The timedomain simulations of Fig. 2 have been performed with $V_{\text {in }}=1.34 \mathrm{~V}$ and a repetition frequency $f_{p}=800 \mathrm{MHz}$, using different values of the pulse width, initially defined as $w_{p}=\alpha / 800 \mu \mathrm{s}$. The input pulse and the voltage at the upper output node (1) have been plotted versus time, considering the interval $80 \mathrm{~ns}$ to $100 \mathrm{~ns}$ in all the cases. For $\alpha=0.31$ [Fig. 2(a)] one obtains a frequency division by two, since the signal period is twice the period of the input pulse. For $\alpha=0.4$ [Fig. 2(b)] the solution period agrees with that of the input signal. For $\alpha=0.5$ [Fig. 2(c)] there is an incommensurable oscillation. Finally, for $\alpha=0.69$ [Fig. 2(d)], there is a frequency division by 2 . In all the cases considered in Fig. 2, the pulse amplitude and repetition rate are the same, which evidences that the stability properties change with the pulse width.

Due to the circuit symmetry, the behavior for $\alpha$ and $1-\alpha$ is approximately the same, though the waveforms at the upper and lower transistor branches are exchanged. That is, voltages and currents at the upper (lower) circuit branch with $\alpha$ are nearly the same as those at the lower (upper) branch with 1 $-\alpha$. Small differences are due to the nonzero rise and decay times of the input pulse. Note that when the rise and decay times are different from zero, the excitation waveforms of the two transistors are different for $\alpha$ and $1-\alpha$. They are not simply exchanged in the two transistor branches, but also have different shapes. It is relevant to note that in the case of instability, and for most of the $\alpha$ values, the waveforms in the upper and lower transistor do not exhibit any symmetries, as gathered from Fig. 2(a) and Fig. 2(d). The reason for this has been studied in Fig. 3, which shows the drain voltage waveforms obtained for $\alpha=0.31$. This value corresponds to a frequency division by two. The output waveform at the drain node of the upper branch $\left(V_{d 1}\right)$ shows a clear periodicity at $2 / f_{p}$, while this same periodicity is less pronounced in the drain node of the lower branch $\left(V_{d 2}\right)$. In fact, the division originates in the upper branch $\left(V_{d 1}\right)$ and the lower branch $\left(V_{d 2}\right)$ simply responds to this divided signal, in addition to the input signal.

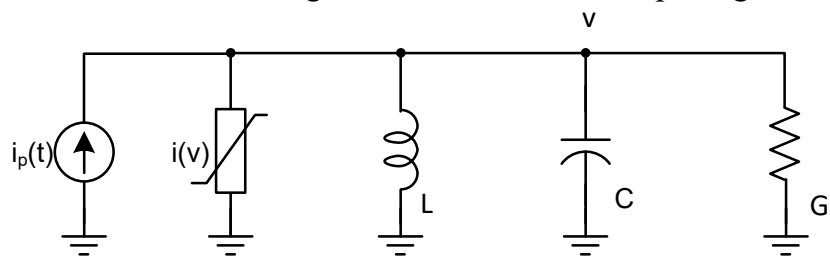

(a)

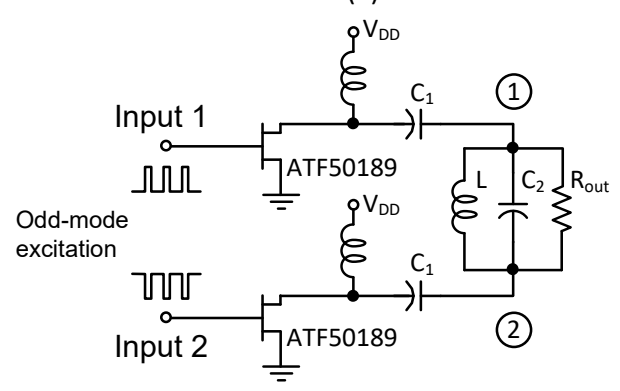

(b)

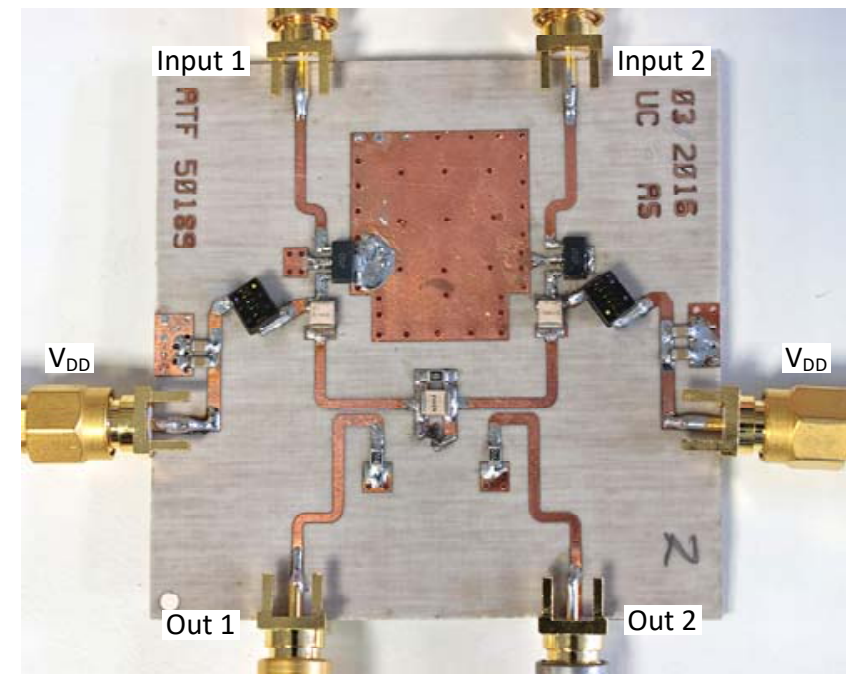

(c)

Fig. 1 Investigation of the instability mechanisms. (a) Resonator circuit with negative-resistance expansion. (b) Proof-of-concept circuit to analyze the influence of the input-pulse width on the stability properties. (c) Photograph of realized circuit (b). 
Actually, for $\alpha=0.31$ the input signal leads the gate voltage of the upper transistor $\left(V_{g 1}\right)$ to a value below the threshold $V_{t o}=0.13 \mathrm{~V}$ for a $69 \%$ of the repetition period $T_{p}$. However, instead of an "off" behavior, a large-amplitude selfoscillation is observed, kicked by the rising edge of the periodic input pulse (see $V_{d 1}$ in Fig. 3). For $\alpha=0.31$ and $V_{i n}=1.34 \mathrm{~V}$, this pulse is able to synchronize the oscillation at one half of the input frequency, so this oscillation exhibits the period $T_{o}=2 T_{p}$. In contrast, the lower transistor (above threshold for most of the repetition period) follows more closely the input source and is not able to self-oscillate. Nevertheless, the solution must be qualitatively the same through the whole circuit, which explains the small amplitude division observed in $V_{d 2}$ of Fig. 3 (small difference in the waveform maxima). Finally, for $\alpha=0.5$, the two amplifier branches exhibit a self-oscillation, which is not synchronized to the input source [Fig. 2(c)].

Note that the assessment of the circuit stability by means of standard time-domain integration relies on an inspection of the waveforms, which can be computationally costly and/or inconclusive in the case of long transients. Moreover, there can be a limited accuracy and/or convergence difficulties. These are the reasons why it could not be applied to the fourstage triband class-S amplifier studied in Section V.

Therefore, in the following, a frequency-domain stability analysis of the class-D circuit in Fig. 1 is presented. It will enable a detailed investigation of the mechanisms and characteristics of instability phenomena in differential circuits driven with a rectangular pulse.

\section{Frequency-domain analysis}

A general frequency-domain formulation, applicable to circuits driven by periodic pulses, is presented, which should provide insight into the effect of the pulsed signal on the stability properties. Note that this pulsed signal constitutes an external input to the nonlinear dynamical system, which actually depends on the circuit components and topology. The periodic pulse is represented in a Fourier series at the fundamental frequency $\omega_{p}$. Using a piecewise formulation [17],[19], the HB system can be expressed as:

$$
A_{k}\left(k \omega_{p}\right) \bar{X}_{k}+B_{k}\left(k \omega_{p}\right) \bar{Y}_{k}(\bar{X})+D_{k}\left(k \omega_{p}\right) \bar{G}_{k}=\overline{0}
$$

where $\omega_{p}$ denotes the fundamental frequency, $k$ is the harmonic order, going from $k=-N H$ to $N H, \bar{X}_{k}, \bar{Y}_{k}(\bar{X})$ and $\bar{G}_{k}$ are the vectors of control voltages of the nonlinear elements, the nonlinear currents and charges, and the input sources, respectively, at the $k^{\text {th }}$ harmonic term, and $\bar{X}$ is the vector containing the full set of harmonic components of the control voltages. The matrices $A_{k}\left(k \omega_{p}\right), B_{k}\left(k \omega_{p}\right), D_{k}\left(k \omega_{p}\right)$ account for the linear passive network, evaluated at the harmonic component $k \omega_{p}$.

With a sinusoidal input signal or signals, all the vectors $\bar{G}_{k}$ at $|k|>1$ are zero, unlike the case of a pulse-train excitation, having harmonic components (included in $\bar{G}_{k}$ ) that depend on the pulse amplitude $V_{i n}$, repetition period $T_{p}$ and duty cycle. This input harmonic content will have an impact on the steady-state solution $\bar{X}_{s}$ both directly and through mixing effects. For the stability analysis of the periodic regime resulting from the pulsed-signal excitation, a small signal perturbation at the complex-frequency $s$ will be considered [13]-[14],[17], so that the nonlinear elements can be linearized about $\bar{X}_{s}$. This provides the following characteristic system:

$$
\left[A\left(\omega_{p}, s\right)+B\left(\omega_{p}, s\right) \partial \bar{Y}\left(\bar{X}_{s}\right) / \partial \bar{X}\right] \Delta \bar{X}=0
$$

where $\Delta \bar{X}$ is the small increment undergone by the circuit variables, and the vector and matrices $\bar{X}, \bar{Y}, A, B$ include all the harmonic indexes $k=-N H$ to $N H$ of those defined in (2). In fact, the matrices $A\left(\omega_{i n}, s\right)$ and $B\left(\omega_{i n}, s\right)$ are block diagonal, composed of submatrices $A_{k}, B_{k}$, in which the circuit linear elements are evaluated at the frequencies $j k \omega_{p}+s$. The Jacobian matrix $\partial \bar{Y}\left(\bar{X}_{s}\right) / \partial \bar{X}$ is made up of the derivatives of the harmonic components of the device nonlinearities with respect to the harmonic components of their control voltages, evaluated at the periodic steady state. The matrix $\partial \bar{Y}\left(\bar{X}_{s}\right) / \partial \bar{X}$ is easily calculated from the Fourier coefficients of the derivatives of the constitutive relationships of the device nonlinearities with respect to their control voltages [13]-[14],[17]. It accounts for nonlinear dynamic elements in the transistor, which would correspond to nonlinear capacitances, described with their harmonic components. The multiplication by terms of the form $j k \omega_{p}+s$, associated with the time-differentiation operation, is performed when doing $B\left(\omega_{p}, s\right) \partial \bar{Y}\left(\bar{X}_{s}\right) / \partial \bar{X}$ [13]-[14],[17],[38]. The time delays inside the transistor are taken into account by evaluating the corresponding delay-dependent exponential functions at the perturbation frequencies $j k \omega_{p}+s$.

The solution poles agree with the roots of the determinant of the matrix affecting $\Delta \bar{X}$ in (3), known as the "characteristic determinant" [13]-[14],[30]. Because the pulsed signal constitutes an external input to the nonlinear dynamical system, the characteristic system in (3) is formally the same as the one obtained under a sinusoidal excitation. For a given harmonic number $N H$, the matrices $A$ and $B$ are independent of the type of periodic excitation (pulse train or sinusoidal), so this excitation affects the stability properties through its impact on the steady-state solution $\bar{X}_{s}$ and the Jacobian matrix $\partial \bar{Y}\left(\bar{X}_{s}\right) / \partial \bar{X}$. At constant $V_{i n}$, and $T_{p}$, more harmonic terms become relevant in $\bar{X}_{s}$ and $\partial \bar{Y}\left(\bar{X}_{s}\right) / \partial \bar{X}$ when decreasing the duty cycle.

At this point, one should take into account that the stability properties of a periodic solution are formally defined by its corresponding finite set of Floquet multipliers [21],[34]-[35]. There are as many multipliers as the dimension of the differential-algebraic-equation system that governs the circuit behavior. This dimension is the same in small- and largesignal conditions, unlike the dimension of the frequencydomain system (3), which changes with $N H$. For a harmonic number $N H$ tending to infinite, the poles $s$ agree with the Floquet exponents [21],[34]-[35]. They are related with the Floquet multipliers in a non-univocal manner, as $m=e^{s T_{p}}$, where $T_{p}$ is the solution period. Therefore, all the poles of the 
form $j k \omega_{p}+s$ should map onto the same Floquet multiplier(s), which may be real and complex-conjugate. This relationship must be kept in mind during the investigation of the stability properties of the class-D configuration.

To get some insight into the circuit behavior under an arbitrary input signal, system (3) can be decomposed into a set of vector equations at the different harmonic terms, in the following manner:

$$
\begin{aligned}
& \left\{\left[A_{0}(s)\right]+\left[B_{0}(s)\right] \partial \bar{Y}_{0}\left(\bar{X}_{s}\right) / \partial \bar{X}_{0}\right\} \Delta \bar{X}_{0}+ \\
& {\left[B_{0}(s)\right] \sum_{m \neq 0,-N H}^{N H} \partial \bar{Y}_{0}\left(\bar{X}_{s}\right) / \partial \bar{X}_{m} \Delta \bar{X}_{m}=0,} \\
& {\left[A_{k}\left(j k \omega_{i n}+s\right)\right] \Delta \bar{X}_{k}+\left[B_{k}\left(j k \omega_{i n}+s\right)\right] \sum_{m=-N H}^{N H} \partial \bar{Y}_{k}\left(\bar{X}_{s}\right) / \partial \bar{X}_{m} \Delta \bar{X}_{m}=0,}
\end{aligned}
$$

where $k \neq 0$

(4)

where the equation corresponding to $k=0$ has been separated from the rest and $k$ in the lower expression goes from $-\mathrm{NH}$ to $N H$, except $k=0$. The equations are coupled through the dependence of $\bar{Y}_{k}$ on $\bar{X}_{m}$, where $m \neq k$. For low input amplitude, the matrices $\partial \bar{Y}_{k}\left(\bar{X}_{s}\right) / \partial \bar{X}_{m}$, where $k \neq m$, will be also be small, as is easily derived from the fact that they correspond to the harmonic components $k-m$ of the time varying Jacobian matrix $\partial \bar{y}\left(\bar{x}_{s}(t)\right) / \partial \bar{x}$, where $\bar{x}_{s}(t)$ is the periodic steady-state solution in time domain. In the usual case of a sinusoidal excitation (in a dc-decoupled circuit), the dc vector $\bar{G}_{0}$ is independent of the driving source(s). However, in the case of a rectangular pulse, it will depend on $V_{\text {in }}, T_{p}$ and $w_{p}$, which will affect the dc term $\bar{X}_{0}$, having a relevant impact on the stability properties. For larger input amplitude, the behavior will be consistent with the continuity of the circuit equations, so there will be a continuous variation of the poles, versus this amplitude, in agreement with the discussion in Section II.A.

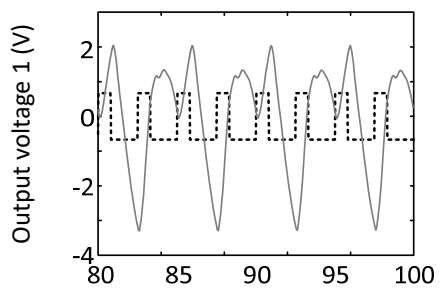

(a)

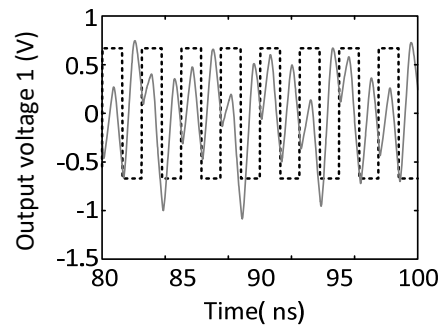

(c)

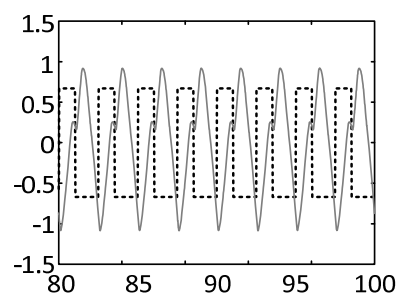

(b)

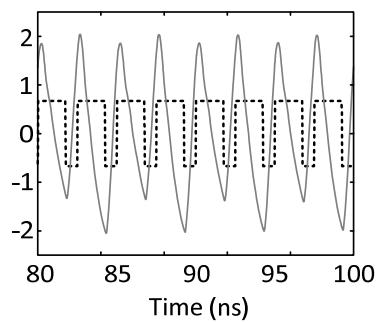

(d)
Fig. 2 Time-domain analysis of the circuit in Fig. 1(b) for different values of the pulse width. The pulse repetition frequency is $f_{p}=800$ $\mathrm{MHz}$ and the total amplitude is $V_{i n}=1.34 \mathrm{~V}$. The pulse width is defined as $w_{p}=\alpha / 800 \mu$ s. (a) Case $\alpha=0.31$, frequency division by two. (b) Case $\alpha=0.4$, the solution period agrees with that of the input signal. (c) Case $\alpha=0.5$, incommensurable oscillation. (d) Case $\alpha=0.69$, frequency division by two.

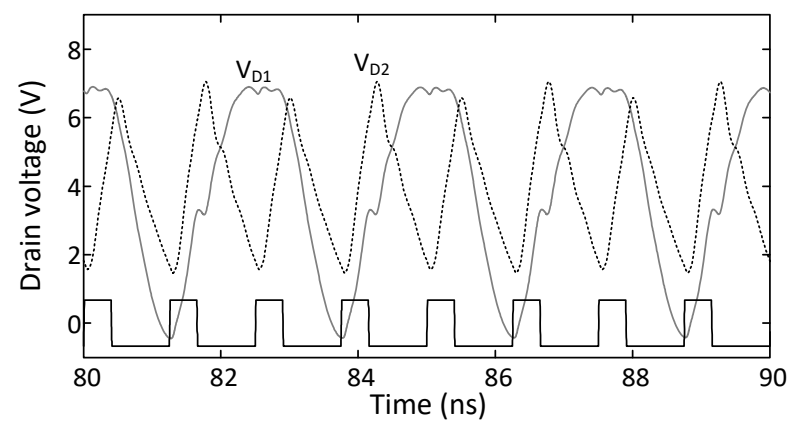

Fig. 3 Drain voltage waveforms $\left(V_{D 1}\right.$ and $\left.V_{D 2}\right)$ of the two transistors in the circuit of Fig. 1(b) for $\alpha=0.31 . V_{D 1}$ is in solid line and $V_{D 2}$ is in dotted line. The input pulse at $f_{p}=800 \mathrm{MHz}$ is shown at the bottom.

\section{Application to the class-D configuration}

In practice, the poles will be analyzed with pole-zero identification [11]-[12],[35]. This requires the calculation of a closed-loop transfer function by introducing a small-signal source at an incommensurate frequency $\Omega$. Advantage is taken from the fact that all the possible transfer functions share a same denominator, which agrees with the characteristic determinant, evaluated at $j \Omega$ instead of $s$. To obtain the transfer function, a small-signal current source $I_{s}$, at a frequency $\Omega$ incommensurable with the frequency of the input pulse $\omega_{p}$, is introduced into the circuit [11]-[12],[35]. Then, the closed loop transfer function is calculated as the ratio between the voltage $V_{s}$ at the source connection node and the current introduced: $Z(\Omega)=V_{s} / I_{s}$. This complex function is fitted with a quotient of polynomials externally to the harmonicbalance software. As stated, the denominator of all the possible transfer functions should be the same, regardless of the particular location of the current source, but the numerator will change depending on this location. It is recommended to choose observation nodes in the immediate neighborhood of the intrinsic device terminals, as these will enable the highest sensitivity to the possible instability mechanisms.

Regarding the $\Omega$ interval considered for the pole-zero identification, one should take into account the non-univocal relationship between the Floquet exponents (or poles) and the Floquet multipliers [34]-[35], discussed later in more detail. This implies that the same stability information should be obtained in any frequency interval $k \omega_{i n},(k+1) \omega_{i n}$, where $k$ is an integer. In practice, and due to the numerical limitations, there can be differences in the analysis sensitivity. Here we have used the interval $\left(0, \omega_{i n}\right)$, comprising the dominant poles detected in the stability analysis of the dc solution.

This analysis has been applied to the test-bench circuit in Fig. 1(b-c), considering the input frequency $f_{p}=800 \mathrm{MHz}$, input amplitude $V_{\text {in }}=1.34 \mathrm{~V}$ and varying the pulse width from $\alpha=0.1$ to $\alpha=0.9$. The small signal current source $I_{s}$ has been connected to the transistor gate and drain terminals, obtaining equivalent results. Fig. 4 presents these results in the most 
significant interval, going from $\alpha=0.25$ to $\alpha=0.75$. For most of the $\alpha$ values, there are two different pairs of dominant complex-conjugate poles, one at an incommensurable frequency $f_{a}$ and the other at about the subharmonic frequency $f_{p} / 2$, where $f_{p}$ is the pulse repetition rate. Depending on the $\alpha$ value, either one pair or the other becomes the critical one, passing the other, and even crossing to the RHS. This is due to the changes in the average value of the input pulse, shown in the upper horizontal axis of Fig. 4(a). The intricate behavior will be explained after an analysis of the circuit stability properties in dc regime.

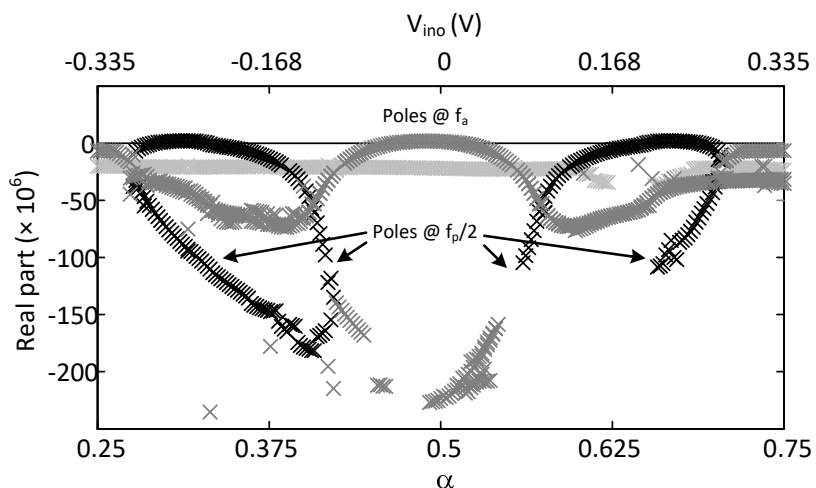

(a)

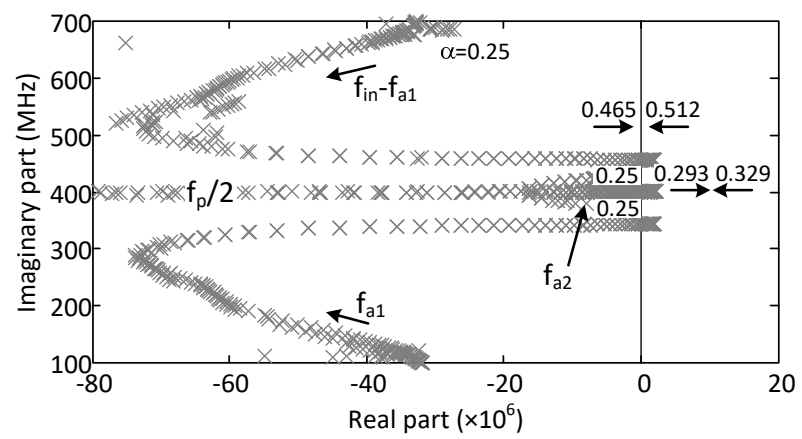

(b)

Fig. 4 Stability analysis of the circuit in Fig. 1(b), performed for a constant pulse repetition frequency $f_{p}=800 \mathrm{MHz}$ and a constant amplitude $V_{\text {in }}=1.34 \mathrm{~V}$, versus the pulse width, expressed as $w_{p}=\alpha / 800 \mu \mathrm{s}$. The upper horizontal axis indicates the average value of the input pulse.

The pulsed source is replaced by two de voltage sources of values $V_{\text {ino }}$ and $-V_{\text {ino }}$, each connected to one of the two circuit branches. Then, a small-signal stability analysis (of the dc solution) is carried out versus the dc voltage $V_{i n o}$. The results are shown in Fig. 5, where the real part of the dominant poles has been represented. The circuit is stable for all the $V_{\text {ino }}$ values. As expected, and due to the symmetry in the circuit topology, the behavior is symmetric with respect to $V_{\text {ino }}=0 \mathrm{~V}$. Around $0 \mathrm{~V}$, the dominant pair of complex-conjugate poles has a frequency of about $368 \mathrm{MHz}$. In the intermediate region, $-0.13 \mathrm{~V} \leq V_{\text {ino }} \leq 0.13 \mathrm{~V}$, both transistors are biased below threshold, so the two circuit branches contribute to the characteristic system (and its natural frequencies) in a nearly odd-symmetrical manner. At $V_{\text {ino }}= \pm 0.13 \mathrm{~V}$, agreeing with the threshold $V_{t o}=0.13 \mathrm{~V}$, the pair of complex-conjugate poles at about $368 \mathrm{MHz}$ moves away from the imaginary axis (and decreases in frequency towards $95 \mathrm{MHz}$ ) very quickly.
For $\left|V_{\text {ino }}\right|>0.13 \mathrm{~V}$, a different pair of complex-conjugate poles, at about $404 \mathrm{MHz}$, approaches the imaginary axis. One of the transistors is below threshold and the other is above threshold, which significantly affects the roots of the characteristic determinant through its impact on the Jacobian matrix $\partial \bar{y}\left(\bar{x}_{d c}\right) / \partial \bar{x}$, where $\bar{x}_{d c}$ is the dc solution.

The pole locus in Fig. 4 (obtained in the presence of the periodic input pulse) evidences the existence of two distinct dominant pairs of poles, in agreement with the small-signal stability analysis in Fig. 5. Depending on the particular $\alpha$ interval, the input pulse pushes either one pair or the other to the RHS. For each $\alpha$ value, the critical pair of poles roughly agrees with the one obtained in dc regime for the average value of the input pulse, that is, for $V_{\text {ino }}=1 / T_{p} \int v_{p}(t) d t$. This is due to the relatively small value of the input amplitude, $V_{i n}=1.34 \mathrm{~V}$. See the upper horizontal axis of Fig. 4 (a) and compare with the results in Fig. 5. Note that the existence of two different pairs of critical poles is not peculiar to this circuit, since it is due to the biasing effect of the average value of the input pulse. Of course, dynamical effects coming from the time variation of the input signal have an impact on the intervals for which each of the two pairs is the critical one, so predictions based on the dc analysis in Fig. 5 are only approximate. As in the two simple cases discussed in $A$, the time-varying input signal is able to shift the original circuit poles (at the resonance frequencies obtained for $V_{\text {ino }}$ ) to the RHS.

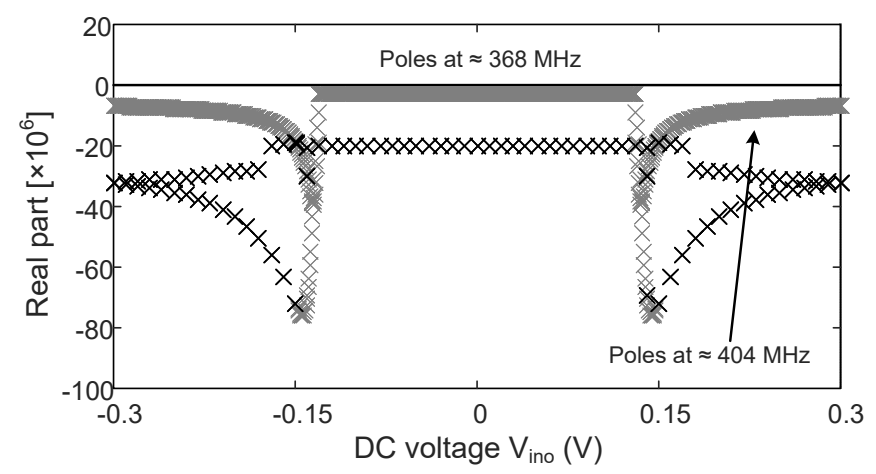

Fig. 5 Poles of the dc solution when replacing the pulsed input source with two de voltage sources of values $V_{\text {ino }}$ and $-V_{\text {ino. }}$ The real part of the dominant poles has been traced versus $V_{\text {ino }}$.

To understand the pole evolution in Fig. 4, one must take into account the non-univocal relationship between the Floquet multipliers [34]-[35] and poles of a periodic solution [17], which has the form $m=e^{s T_{p}}$. If the frequency $f_{a}$ of a particular pair of complex conjugate poles $\left(\sigma \pm j 2 \pi f_{a}\right)$ is incommensurable with $f_{p}$, together with this pair of poles, there will be a whole set of poles of the form $\sigma \pm j 2 \pi\left(f_{a}+k f_{p}\right)$, where $k \neq 0$ is an integer number. As gathered from the relationship $m=e^{s T_{p}}$, all these poles give rise to a same pair of complex-conjugate Floquet multipliers $m_{ \pm}=e^{\left(\sigma \pm j 2 \pi f_{a}\right) T_{p}}$, so they will be denoted here "associated poles". For convenience, Table I summaries the relationship between the 
the Floquet multipliers and the lowest-frequency "associated poles".

Next, the evolution of the circuit stability properties when varying $\alpha$ at the constant input amplitude $V_{\text {in }}=1.34 \mathrm{~V}$ and frequency $f_{p}=800 \mathrm{MHz}$ will be investigated. For convenience, Table II indicates the main different stability situations obtained when varying $\alpha$. For $\alpha=0.25$ [Fig. 4(b)], there are two independent families of dominant complex-conjugate poles, at two different frequencies $f_{a 1}$ (evolving from 100 $\mathrm{MHz}$ to about $370 \mathrm{MHz}$ ) and $f_{a 2}$ (about $400 \mathrm{MHz}$ ). Each of these two independent families of poles is associated with a different pair of complex-conjugate multipliers, $m_{ \pm}^{1}$ and $m_{ \pm}^{2}$. In particular, the pairs of complex-conjugate poles at $f_{a}$ and $f_{p}-f_{a}$ of each of the two families have been represented in Fig. 4(b). Remember that the input-pulse frequency is $f_{p}=800$ MHz. The two different $f_{a}$ frequencies are $f_{a 1}=100 \mathrm{MHz}$ (and, thus, $f_{p}-f_{a 1}=700 \mathrm{MHz}$ ) and $f_{a 2}=390 \mathrm{MHz}$ (and, thus, $f_{p}-f_{a 2}$ $=410 \mathrm{MHz}$ ). The two associated pairs of complex-conjugate poles (at $f_{a}$ and $f_{p}-f_{a}$ ) are clearly distinguished from the inspection of Fig. 4(b), since they have the same real part $\sigma$. The poles at $f_{a 2}=390 \mathrm{MHz}$ (and $410 \mathrm{MHz}$ ) are closer to the imaginary axis. It is emphasized that each set of two pairs of complex-conjugate poles (at $f_{a}$ and $f_{p}-f_{a}$ ) is associated with a different pair of complex-conjugate multipliers, so there are two different pairs of critical complex-conjugate multipliers, in agreement with the two different pairs of critical poles detected in the dc stability analysis of Fig. 5.

When increasing $\alpha$, the poles at $f_{a 2}$ and $f_{p}-f_{a 2}$ (around $400 \mathrm{MHz}$ ) approach each other until they merge and split into two independent pairs of poles at $f_{p} / 2$. At the merging point, each independent pair of poles at at $f_{p} / 2$ gives rise to one negative real multiplier since $m=e^{\left(\sigma \pm j 2 \pi f_{p} / 2\right) T_{p}} \in R^{-}$. Note that this transformation into two independent real multipliers preserves the dimension of the critical subspace.

Table I Summary of the relationships between the Floquet multipliers and poles

\begin{tabular}{|l|l|l|}
\hline Floquet multipliers & $\begin{array}{l}\text { Critical poles } \\
\text { in the band }\left(0, f_{p}\right)\end{array}$ & $\begin{array}{l}\text { Associated } \\
\text { Bifurcation }\end{array}$ \\
\hline$m_{1} \in R^{+}$ & $\sigma, \sigma \pm j 2 \pi f_{p}$ & Fold \\
\hline$m_{2} \in R^{-}$ & $\sigma \pm j 2 \pi f_{p} / 2$ & Flip \\
\hline $\begin{array}{l}m_{2}, m_{3} \in C \\
m_{2}=\operatorname{conj}\left(m_{3}\right)\end{array}$ & $\sigma \pm j 2 \pi f_{a}, \sigma \pm j 2 \pi\left(f_{p}-f_{a}\right)$ & Hopf \\
\hline
\end{tabular}

At $\alpha=0.293$ one of the pairs of poles at $f_{p} / 2$ crosses the imaginary axis to the RHS in a flip bifurcation [13]-[14],[29] (Table I), giving rise to a frequency division by two. The subharmonic instability persists up to the value $\alpha=0.329$, at which this pair of poles crosses to the left hand side of the complex plane (LHS) (inverse flip bifurcation) and gives rise to stable behavior. As this pair of poles gets away from the imaginary axis, the other pairs of poles, originally at $f_{a 1}=100 \mathrm{MHz}$ (and $f_{p}-f_{a 1}=700 \mathrm{MHz}$ ), approach this imaginary axis, with $f_{a 1}$ increasing quickly, and cross to the RHS at $\alpha=0.465$, at the frequency $340 \mathrm{MHz}$, in a Hopf bifurcation [13]-[14],[29] (Table II). They cross again to the LHS at $\alpha=0.512$ (inverse Hopf bifurcation). As $\alpha$ continues to increase, the behavior is the reverse one, and the poles nearly pass again through the same values [Fig. 4(a)], but in an inverse sense, which is due to the symmetry of the class-D configuration in Fig. 1(b). These results are in full agreement with the time-domain simulations of Fig. 2.

Table II Stability situations at $V_{i n}=1.34 \mathrm{~V}, f_{p}=800 \mathrm{MHz}$

\begin{tabular}{|c|c|c|}
\hline$\alpha$ & $\begin{array}{l}\text { dc absolute value } \\
\left|V_{\text {ino }}\right|(\mathrm{V})\end{array}$ & Stability situation: \\
\hline 0.25 & 0.295 & $\begin{array}{l}\text { Stable. Frequencies of dominant } \\
\text { poles: } \\
f_{a 1}=100 \mathrm{MHz} \\
f_{p}-f_{a 1}=800-100=700 \mathrm{MHz} \\
f_{a 2}=390 \mathrm{MHz} \\
f_{p}-f_{a 2}=800-390=410 \mathrm{MHz}\end{array}$ \\
\hline 0.293 & 0.237 & $\begin{array}{l}\text { Flip bifurcation. Critical poles } \\
\text { crossing to the RHS: } \\
f_{p} / 2=400 \mathrm{MHz}\end{array}$ \\
\hline 0.329 & 0.189 & $\begin{array}{l}\text { Inverse flip bifurcation. Critical } \\
\text { poles crossing to the LHS: } \\
f_{p} / 2=400 \mathrm{MHz}\end{array}$ \\
\hline 0.465 & 0.007 & $\begin{array}{l}\text { Hopf bifurcation. Critical poles } \\
\text { crossing to the RHS: } \\
f_{a 1}=340 \mathrm{MHz} \\
f_{p}-f_{a 1}=800-340=460 \mathrm{MHz}\end{array}$ \\
\hline 0.512 & 0.056 & $\begin{array}{l}\text { Inverse Hopf bifurcation. Critical } \\
\text { poles crossing to the LHS: } \\
f_{a 1} \cong 340 \mathrm{MHz} \\
f_{p}-f_{a 1} \cong 800-340=460 \mathrm{MHz}\end{array}$ \\
\hline
\end{tabular}

To summarize, the circuit in Fig. 1(b) is unstable when there are poles on the RHS. Two different kinds of instability have been detected: frequency division by 2 and incommensurable oscillations. Table I indicates the two types of bifurcation phenomena. The frequency division is due to a flip bifurcation [13]-[14],[29], at which a pair of complexconjugate poles at the subharmonic frequency $\omega_{p} / 2$ cross the imaginary axis, which is observed for $\alpha=0.293$ and $\alpha=0.329$. This phenomenon defines the boundaries of the parameter interval leading to a subharmonic oscillation. On the other hand, the incommensurable oscillation is due to a Hopf bifurcation [13]-[14],[29], at which a pair of complexconjugate poles at an incommensurable frequency, $\omega_{a}=2 \pi f_{a}$, cross the imaginary axis, which is observed for $\alpha=0.465$ and $\alpha=0.512$. The possibility to detect these bifurcations, in a circuit excited with a pulsed signal, will be demonstrated in the next section.

\section{GLOBAL STABILITY ANALYSIS}

In practical applications, the circuit is expected to operate under different values of the pulse amplitude, repetition frequency and pulse width. Therefore, it will be interesting to 
obtain the stability boundaries under variations of these parameters in an efficient manner. This will be done through a bifurcation-detection technique based on the use of auxiliary generators [17],[28] and fully compatible with the use of commercial HB. For completeness, a brief contextualization of this method is presented next.

\section{A. Bifurcation-analysis methods}

Most numerical methods for bifurcation detection in the nonlinear dynamics field [20], [22], [39], [39]-[40] depart from an explicit description of the nonlinear differential equation system in time domain. Methods like those based on the Poincaré map or the direct calculation of Floquet multipliers [21]-[22], [29] are intended for a time-domain description of the nonlinear circuit. In the frequency domain, when having access to the $\mathrm{HB}$ equation system and its Jacobian matrix [13]-[17], as in in-house software, the bifurcation detection is usually based on the calculation of the parameter values at which the characteristic determinant, associated with the characteristic system (3), fulfils certain mathematical conditions. In the cases of the Hopf and flip bifurcation, the determinant must be singular at an incommensurable perturbation frequency and subharmonic frequency, respectively [13]-[17]. Clearly, this method is not compatible with commercial HB, since the user does not have access to the HB system and the Jacobian matrix $\partial \bar{Y}\left(\bar{X}_{s}\right) / \partial \bar{X}$. A different methodology is based on the introduction of an auxiliary generator (AG) into the circuit [17], which must fulfil certain mathematical conditions at the bifurcation points [17], [27]. In commercial software this is achieved through optimization, with the whole set of HB equations (transparent to the user) constituting an inner tier.

\section{B. Calculation of the stability boundaries}

The two main types of bifurcations from periodic regime are flip and Hopf bifurcations [13]-[17], [22], [29], [39]-[41]. For their detection, it will be taken into account that, in a steady-state oscillation, the total admittance-function is equal to zero at any circuit node and the oscillation amplitude must tend to zero at the stability boundaries [17].

\section{a) Flip bifurcation}

At the flip bifurcation a subharmonic oscillation of amplitude tending to zero arises or vanishes, this depending on the sense of variation of the analysis parameter. The flip bifurcation can be detected introducing a small-signal current source $I_{s}$ at the subharmonic frequency $f_{p} / 2$ into the circuit, [17]. This small-signal source will be connected in parallel at a sensitive circuit node, such as a device terminal. The limit condition for the subharmonic oscillation is $Y=0$, where $Y$ is the ratio between the current and voltage of the small-signal source. Because the oscillation frequency $f_{p} / 2$ and the pulse repetition frequency $f_{p}$ are harmonically related, the phase $\phi$ of the small-signal source, or, alternatively, the phase of the input source (when the phase of $I_{s}$ is arbitrarily set to zero) will be an unknown of the problem [17]. Considering any two analysis parameters, such as $\alpha$ and $V_{i n}$, the flip-bifurcation locus will be obtained by solving:

$$
Y\left(\phi, \alpha, V_{i n}\right)=0
$$

with the pure harmonic-balance system as an inner tier. Because of the small-signal operation of current source, the above calculation is not numerically expensive.

\section{b) Hopf bifurcation}

In the case of a Hopf bifurcation, the frequency $f_{a}$ of the oscillation that arises at the instability boundary is not harmonically related with the frequency of the input source $f_{p}$ [17]. The Hopf bifurcations can be detected introducing a small-signal current source $I_{s}$ at the incommensurable frequency $f_{a}$ into the circuit [17]. Defining the admittance $Y$ as the ratio between the current and voltage of the small-signal source, the Hopf-bifurcation condition, to be resolved with the conversion matrix-approach, is given by:

$$
Y\left(f_{a}, \alpha, V_{i n}\right)=0
$$

Note that the oscillation frequency, $f_{a}$, which is autonomously generated, varies through the Hopf locus. As already stated, the above complex equation is solved with a large-signal small-signal analysis (conversion-matrix approach), so the calculation is not numerically expensive.

The bifurcation loci obtained in terms of the pulse width (defined by the parameter $\alpha$ ) and the input amplitude $V_{\text {in }}$ are shown in Fig. 6(a). There is a Hopf-bifurcation locus, located in the center of the diagram, and two flip bifurcation loci, which are nearly symmetrical about $\alpha=0.5$. This symmetrical behavior agrees with the observations in Fig. 2 and Fig. 4, which, as already discussed, is due to the symmetry in the circuit topology. This circuit is unstable inside the Hopf locus and inside the two flip loci. The stability boundaries predicted by pole-zero identification are superimposed with asterisks in Fig. 4, with full agreement.

For too low or too high $\alpha$ there are no instabilities. When reducing the duty cycle, there is a lower threshold value from which onwards the transistor responsible for the oscillation (which, as shown in Fig. 3, is the one driven with the shortest duty cycle) conducts for too short a time. As a result, it is not able to sustain the oscillation. When increasing the duty cycle beyond a certain threshold, the situation is analogous in the other transistor branch, where the transistor is driven with the shortest duty cycle.

The unstable region about $\alpha=0.5$ is delimited by the Hopf locus. Within the entire region, the average value of the pulse is below $V_{t o}$ for the two transistors. As shown in Fig. 5, in this situation the dominant poles of the dc solution have a frequency around $368 \mathrm{MHz}$, too far from $f_{p} / 2=400 \mathrm{MHz}$. At the outermost left and right points of the Hopf locus (corresponding to an input amplitude $V_{\text {in }} \cong 0.5 \mathrm{~V}$ ), the pulse average values, agrees with $V_{t o}=0.13 \mathrm{~V}$. The oscillation frequency varies between $333.4 \mathrm{MHz}$ and $345.8 \mathrm{MHz}$ through the Hopf locus. In general, oscillations are more easily extinguished in an asynchronous manner, which explains the upper closing of the Hopf locus for relatively small values of 
the pulse amplitude $V_{\text {in }}$. In two $\alpha$ intervals (around 0.3 and 0.7), when increasing the input amplitude [Fig. 6(a)] the circuit initially becomes unstable through a Hopf bifurcation (due to an average value below $V_{t o}$ in the two transistors), then it stabilizes through an inverse Hopf bifurcation and becomes unstable again through a flip bifurcation (due to average values above and below $V_{\text {to }}$ in the two transistors). The Hopf instability should not be a problem under normal operation of the class-D configuration, but will arise during testing, when increasing the amplitude of the input pulse from a low value.

The subharmonic oscillations obtained for moderately low and high $\alpha$ can be related to the poles obtained for the corresponding average values $V_{\text {ino }}$, which have a frequency around $405 \mathrm{MHz}$, quite close to $f_{p} / 2=400 \mathrm{MHz}$. Under the dynamical effects of the input pulse, the frequency of the dominant poles is shifted to $f_{p} / 2$ when increasing $V_{\text {in }}$ and they cross to the RHS when crossing the flip-bifurcation locus. The subharmonic instability requires higher input amplitude, which can be related to the original situation in the dc stability analysis (Fig. 5), where the poles around $405 \mathrm{MHz}$ are more distant to the imaginary axis than the poles around $368 \mathrm{MHz}$.

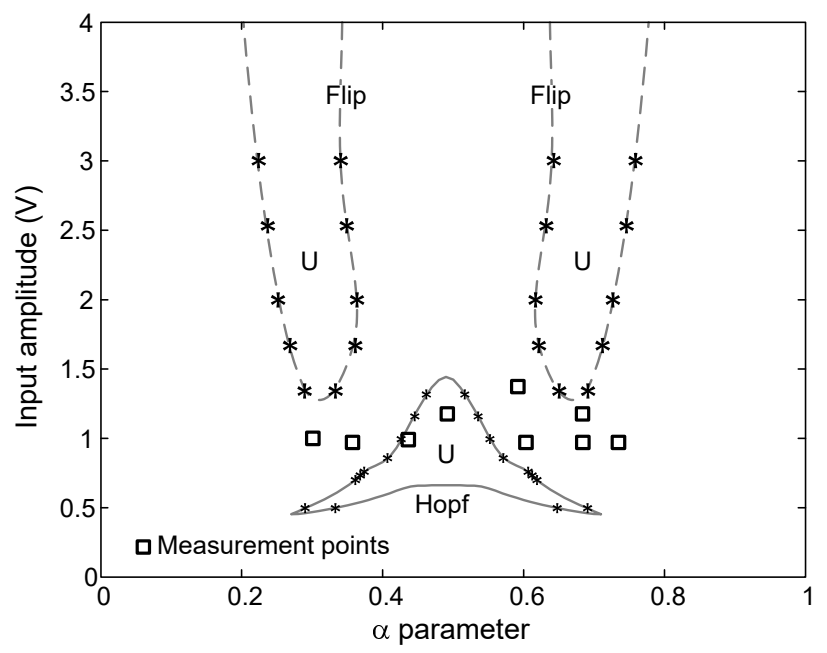

(a)

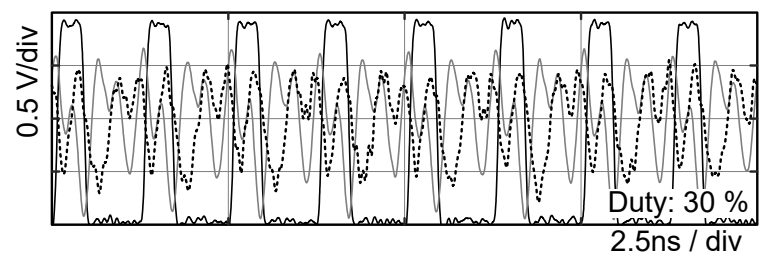

(b)

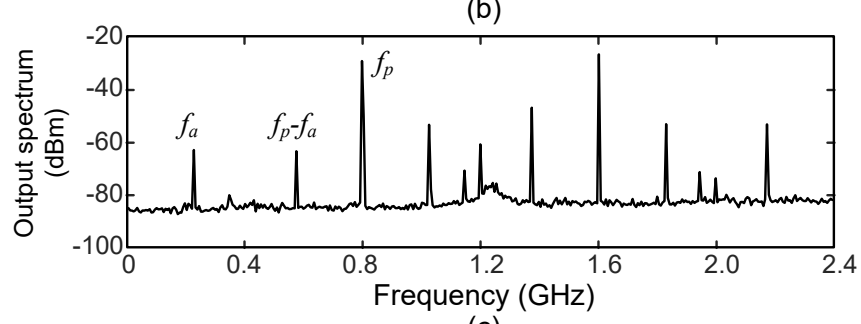

(c)

Fig. 6 Instability at $f_{p}=800 \mathrm{MHz}$. (a) Bifurcation loci in the plane defined by the parameter $\alpha$ and the input amplitude $V_{\text {in }}$. The circuit is unstable inside the flip and Hopf bifurcation loci. Stability boundaries obtained with pole-zero identification are indicated with asterisks. "U" stands for unstable. Measurement points obtained with the set-up in Fig. 7 are superimposed. (b) and (c) Measured waveforms and spectrum for $V_{i n}=1 \mathrm{~V}$ and $\alpha=0.3$, corresponding to a self-oscillating regime. Note that the output signal is highly attenuated by the coupled-line couplers.

\section{Experimental validation}

The circuit has been manufactured [Fig. 1(c)] and experimentally characterized. A photograph of the measurement set-up is shown in Fig. 7. The two input signals (direct and inverted) are obtained with the 81134A Pulse/Pattern Generator from Keysight Technologies, which enables the variation of the duty cycle of the periodic input pulses. The output signals at nodes 1 and 2 in Fig. 1(b) are extracted with coupled-line couplers, connected to an Infiniium 9000 digital sampling oscilloscope. Using a directional coupler, one of the outputs is also measured in a spectrum analyzer, as shown in Fig. 7. At the pulse repetition rate $f_{p}=800 \mathrm{MHz}$ and up to an input amplitude of $2 \mathrm{~V}$, the experimental boundary of the unstable region is given by the measurement points superimposed in Fig. 6(a). In all the cases, the undesired behavior corresponds to a self-oscillating mixer regime, delimited by Hopf bifurcations.

The shape of the experimental Hopf locus approximately agrees with the one predicted in simulation, although it is shifted to higher input amplitudes. The asymmetry of the locus is more pronounced than the one obtained in simulation. This is attributed to the larger values of the pulse rise and decay times. On the other hand, at the frequency considered in Fig. $6\left(f_{p}=800 \mathrm{MHz}\right)$, no frequency divisions could be measured. This is consistent with the upward shift of the Hopf locus. Note that the amplitude of the experimental driving pulse was limited to $V_{\text {in }}=2 \mathrm{~V}$, so we could not use higher $V_{\text {in }}$ values. We have made extensive tests to identify the origin of the discrepancies. Under reasonable tolerance variations of the output circuit elements and choke, the shape of the Hopf locus changed but it was still located in the same amplitude region. However, under an increase of the device output capacitance, there was an upwards shift of both the Hopf and flip loci. In our particular packaged transistor this capacitor might be larger than the one provided in the manufacturer model.

We believe that the deviation is acceptable in a hybrid circuit with packaged transistors. On the other hand, a total correspondence has been found between three independent analyses: pole-zero identification, time-domain integration and bifurcation detection, which strongly suggests that the problem is due to inaccuracies in the component models. As shown in the next section, frequency divisions were indeed measured for lower input frequencies, so the occurrence of the two different phenomena has been experimentally confirmed.

Fig. 6(b) shows the measured waveforms and spectrum for $V_{\text {in }}=1 \mathrm{~V}$ and $\alpha=0.3$, near the edge of the Hopf locus. Note that the output signal is highly attenuated by the coupled-line coupler. Both the spectrum and waveform evidence a selfoscillating mixer regime, in agreement with the predictions of Fig. 6(a) for the lower input amplitudes. As will be shown in the next section, much better agreement between simulation 
and measurements is obtained for lower input frequencies, which is attributed to a smaller impact of parasitic elements in the component models.

\section{BIFURCATIONS ANALYSIS VERSUS THE PULSE REPETITION PERIOD AND PULSE WIDTH}

Because of the possible modulation of the input pulse, obtaining the stability boundaries in the plane defined by the pulse repetition rate and the pulse duration can be of interest. Indeed, a pulse width modulation will only change the original (non-modulated) stability properties from certain value of the pulse-modulation frequency [31].

\section{A. Bifurcation analysis}

Fig. 8(a) presents the Hopf and flip bifurcation loci in the plane defined by $T_{p}$ and $w_{p}$, respectively corresponding to the pulse-repetition period and the pulse time width. The input pulse amplitude is fixed to $V_{i n}=1 \mathrm{~V}$. Keeping in mind the above mentioned effect of the modulation signal, the loci in Fig. 8(a) would be useful for a preliminary prediction of the stability properties in two main cases: a pulse-width modulation with a constant repetition rate and a pulse modulation, with time-varying period. In the first case, and for each repetition period $T_{p}$, the stability properties can be predicted by considering a vertical line at the particular constant value of $T_{p}$. In the second case, and for each pulse width $w_{p}$, the stability properties can be predicted by considering a horizontal line at the particular constant value of $w_{p}$.

For low period $T_{p}$ the stable operation ranges are delimited by the Hopf-bifurcation locus, traced in solid line in Fig. 8(a). For all the operation points composing this locus, there is a pair of critical pair of complex-conjugate multipliers fulfilling $\left|m_{1,2}\right|=1$. Taking into account the non-univocal relationship between the Floquet multipliers and the poles [34]-[35], one can base the analysis on the pairs of complex-conjugate poles $\pm j 2 \pi f_{a}$ and $\pm j 2 \pi\left(f_{p}-f_{a}\right)$, as done in the pole analysis of Fig. 4 . These two pairs of poles are associated with the same critical pair of complex-conjugate multipliers, fulfilling $\left|m_{1,2}\right|=1$.

The frequency $f_{a}$ is incommensurable with $f_{p}$, except at the codimension-two bifurcations discussed below.

The two pairs of poles $\pm j 2 \pi f_{a}$ and $\pm j 2 \pi\left(f_{p}-f_{a}\right)$ can equally be expressed as $\pm j\left(\omega_{p} / 2-\Omega\right)$ and $\pm j\left(\omega_{p} / 2+\Omega\right)$, where $\omega_{p}=2 \pi f_{p}$. The incommensurable offset frequency $\Omega$ varies through the Hopf locus and becomes zero at the four codimension-two bifurcation points, where the flip and Hopf loci merge [see Fig. 8(a)]. These co-dimension-two bifurcations are easily identified in Fig. 8(a). Two of them correspond to the two points where each of the two flip loci (both traced in dashed line and dash-dotted line) merges with the Hopf locus (in solid line), on the left side of the diagram. The other two co-dimension two bifurcations correspond to the edges of the small Hopf-locus section (traced in a bolder solid line) on the right side. This small Hopf-locus section closes the central unstable region by connecting the two flip

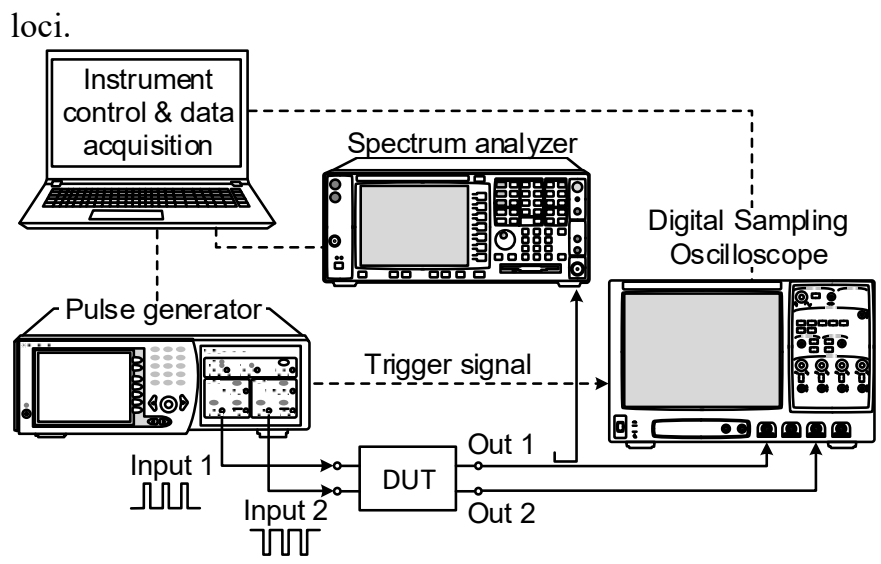

(a)

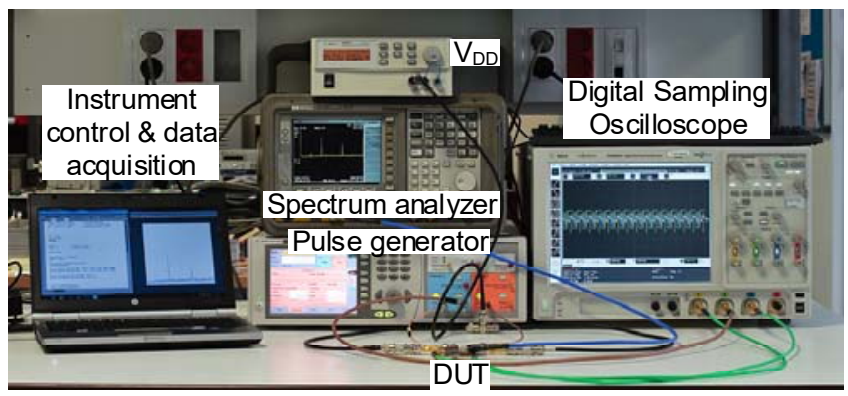

(b)

Fig. 7 Sketch and photograph of the measurement set-up. The two input signals (direct and inverted) are obtained with the $81134 \mathrm{~A}$ Pulse/Pattern Generator from Keysight Technologies, which enables the variation of the duty cycle of the periodic input pulses. The output signals at nodes 1 and 2 in Fig. 1(b) are extracted with coupled-line couplers, connected to an Infiniium 9000 digital sampling oscilloscope and a spectrum analyzer.

The co-dimension-two bifurcations require the fine tuning of two circuit parameters [29],[39]-[41]. When arising from periodic regime, they are characterized by the existence of two independent real Floquet multipliers of magnitude equal to 1 . In this particular case, and for the four co-dimension-two bifurcations, the critical poles degenerate into two independent pairs of poles at $\pm j \omega_{p} / 2$ when $\Omega=0$. When this happens, the critical pair of complex-conjugate multipliers fulfilling $\left|m_{1,2}\right|=1$ (existing through the whole Hopf locus) degenerate into two negative real multipliers of value $m_{1}=m_{2}=-1$ [29]. The degeneracy into two independent real multipliers is consistent with the preservation of the dimension of the critical subspace. From the point of view of the poles, the incommensurable frequency $\omega_{a}$ degenerates into $\omega_{p} / 2$, which gives rise to two independent pairs of complexconjugate poles at $\omega_{p} / 2$, denoted as $\mathrm{p}_{1 / 2}(1)$ and $\mathrm{p}_{1 / 2}(2)$.

At each of the four co-dimension-two bifurcations, there is, in fact, a merging to three curves, one corresponding to the Hopf locus and the other two corresponding to two different sections of a same flip-bifurcation locus. Each flip locus is composed of parameter values for which one of the independent pairs of poles at $\pm j \omega_{p} / 2$ [either $\mathrm{p}_{1 / 2}(1)$ or 
$\mathrm{p}_{1 / 2}(2)$ ] is located on the imaginary axis. Only at the codimension two bifurcations, the two independent pairs of poles $\left(\mathrm{p}_{1 / 2}(1)\right.$ and $\mathrm{p}_{1 / 2}(2)$ at $\left.\omega_{p} / 2\right)$ are simultaneously located on the imaginary axis. When moving away from the codimension-two bifurcation, one of these two pairs of poles at $\omega_{p} / 2$ will shift either to the LHP or to the RHP, this depending on the sense of variation of the parameters from the codimension two bifurcation and through the flip locus.

Inside the two flip-bifurcation loci of Fig. 8(a) (delimited by the dashed line and dash-dotted line sections) the first pair of poles at the subharmonic frequency $\omega_{p} / 2$ [denoted as $\left.\mathrm{p}_{1 / 2}(1)\right]$ is located on the RHP, so the two inner regions of these loci are unstable. In these same regions, the other pair of poles [denoted as $\left.p_{1 / 2}(2)\right]$ will be on the LHS. When crossing the dashed line, the pair of poles $p_{1 / 2}(1)$ will cross to the LHS, so when getting out of the two flip loci through the dashed line sections, the circuit becomes stable. These dashed-line sections constitute a physical stability boundary.

In contrast, when traversing the dash-dotted sections, it is the second pair of poles at $\omega_{p} / 2$ [denoted as $\left.\mathrm{p}_{1 / 2}(2)\right]$, which crosses the imaginary axis to the RHS. Before crossing these sections (and inside the two flip loci), the amplifier is unstable with a pair of poles $\left[p_{1 / 2}(1)\right]$ on the RHS at $\omega_{p} / 2$. After crossing these sections, the amplifier is unstable with two independent pairs of poles on the RHS. Initially both of them will be at the frequency $\omega_{p} / 2$, though once inside the unstable region delimited by Hopf-bifurcation locus and the dash-dotted sections of the flip loci, these poles will transform into a pair of complex conjugate poles at $\omega_{a}=2 \pi f_{a}$ (and $\left.\omega_{p}-\omega_{a}\right)$. Hence, the dash-dotted sections do not constitute a physical instability boundary. To summarize, the circuit will be unstable inside the two flip loci and inside the region delimited by the Hopf locus and the dash-dotted sections of the flip loci.

The above results have been validated with pole zero identification [Fig. 9(a) and Fig. 9(b)]. For $T_{p}=2.8$ ns [Fig. 9(b)] there are two regions with subharmonic instability, with a small stable region in-between. For small repetition period $T_{p}$, the input frequency is too high in comparison with the circuit natural frequencies, so the input signal has no capability to shift the stable poles for any value of the pulse width $w_{p}$. On the other hand, for high repetition periods $T_{p}$, changes in the input signal are too spaced in comparison with the circuit own dynamics and the circuit behaves in a stable manner, in consistency with the stability of the dc solution for any $V_{\text {ino. }}$. For a ratio $w_{p} / T_{p}$ about 0.5 (straight line in Fig. 8), the circuit becomes unstable only through Hopf bifurcations, obtained for the higher repetition frequencies. Subharmonic instabilities are obtained in both the upper and lower sections, which is in agreement with the symmetry properties observed in Fig. 4 to Fig. 6.

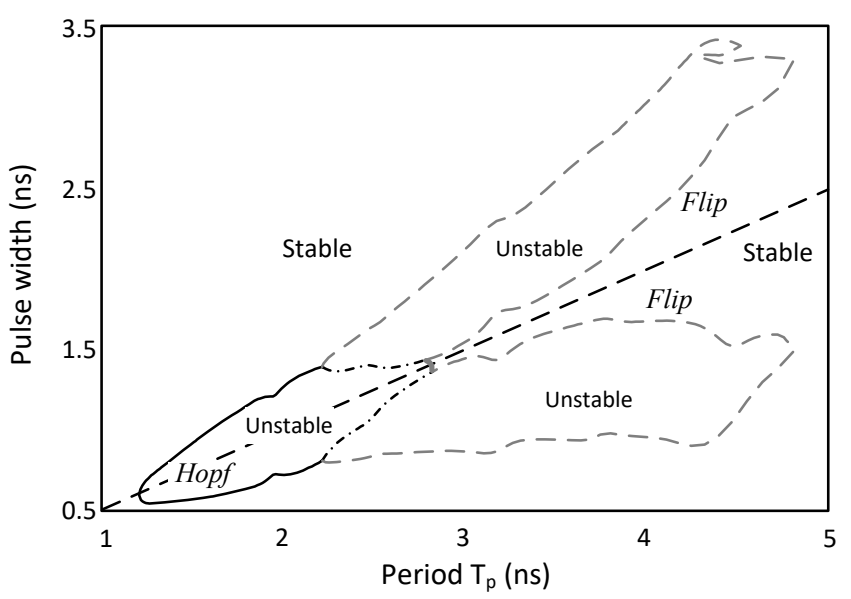

(a)

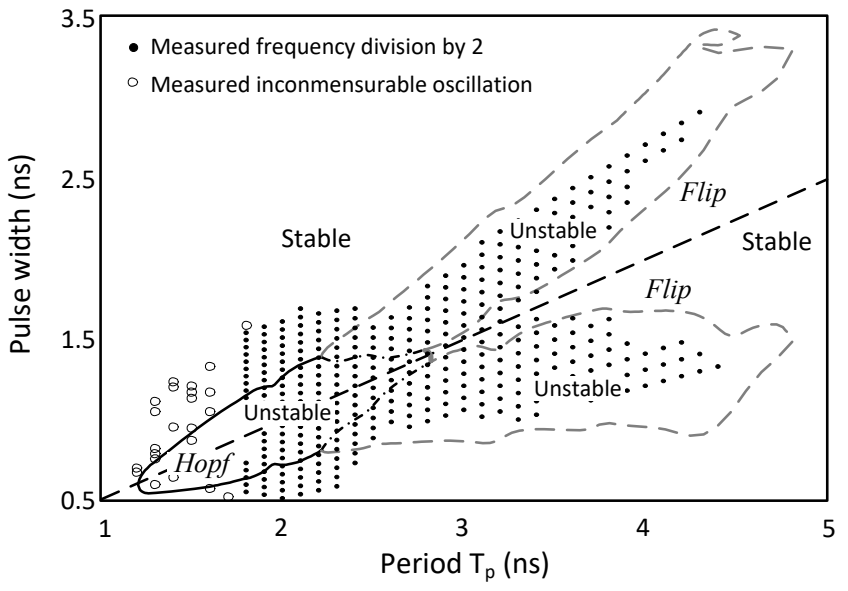

(b)

Fig. 8 Stability analysis versus $T_{p}$ and $w_{p}$. The input pulse amplitude is fixed to $V_{i n}=1 \mathrm{~V}$. (a) The Hopf and flip bifurcation loci have been traced in the plane defined by $T_{p}$ and $w_{p}$. (b) Experimental validation of the results in (a), showing the pairs of values of the pulse period $T_{p}$ and pulse width $w_{p}$ that lead to undesired behavior. Self-oscillating mixing regimes are indicated with circles. Frequency divisions are indicated with dots.

\section{B. Experimental validation}

The results in Fig. 8(a) have been validated experimentally [Fig. 8(b)] by programming the measurement set-up so as to detect the two types of undesired behavior: self-oscillating mixing regime, when the oscillation frequency is incommensurable with that of the input pulse, and subharmonic regime, when the oscillation frequency agrees with the input frequency divided by two. The first type of operation is indicated with circles. The second type of operation is indicated with dots. In Fig. 8(b) results have been superimposed on the Hopf and flip bifurcation loci of Fig. 8(a), with good agreement. Measurements validate the nearly symmetrical behavior about the straight line corresponding to a $50 \%$ duty cycle, as well as the presence of Hopf bifurcations only for the smaller values of the input-pulse period $T_{p}$. The fact that the dash-dotted sections of the two flip loci do not constitute a stability boundary is confirmed too, by comparison of Fig. 8(a) and Fig. 8(b).

It is worth mentioning that when moving away from the 
unit-slope straight line, instability occurs only in one of the circuit branches in many cases, as observed in simulation. Furthermore, outside the experimental unstable regions of Fig. 8(b), an oscillation is often measured in the absence of one of the two inputs, but this oscillation is extinguished as soon as the second input is introduced into the circuit. This is attributed to an asynchronous extinction due to the signal injected by the non-oscillatory circuit branch, when driven by the input generator. Fig. 10 shows the spectra obtained for the pulse period $T_{p}=3.33 \mathrm{~ns}$ and the duty cycles $35 \%, 50 \%$ and 65\%, respectively. Measurements in Fig. 10(a) and Fig. 10(c) exhibit a frequency division by two, in full agreement with the predictions of the loci in Fig. 8. In the measurements of Fig. 10 (b) (corresponding to $50 \%$ duty cycle) there are no undesired spectral components, also in agreement with the predictions of Fig. 8.

The behavior under a pulse-width modulation $\alpha(t)=\alpha_{o}+\Delta \alpha \sin \left(2 \pi f_{\text {mod }} t\right)$ have been analyzed with the envelope-transient method [32], expressing the input signal in a Fourier series with harmonic terms $\bar{G}_{k}(t)$, varying in time at the rate of the modulation. For low $f_{\text {mod }}$ the circuit behaves in an unstable manner if $\alpha(t)$ enters the unstable regions obtained in the time map of Fig. 8. When the modulation frequency increases towards the order of $\mathrm{MHz}$, the circuit becomes stable in all the cases tested, even when entering the unstable regions of Fig. 8, in agreement with [31].

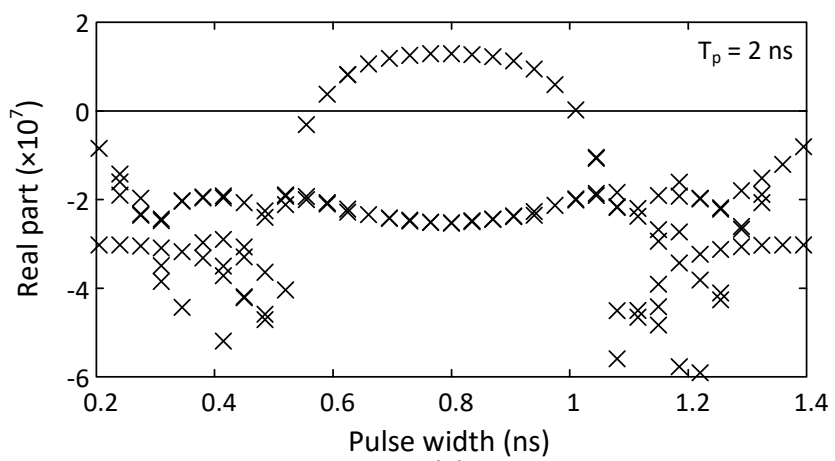

(a)

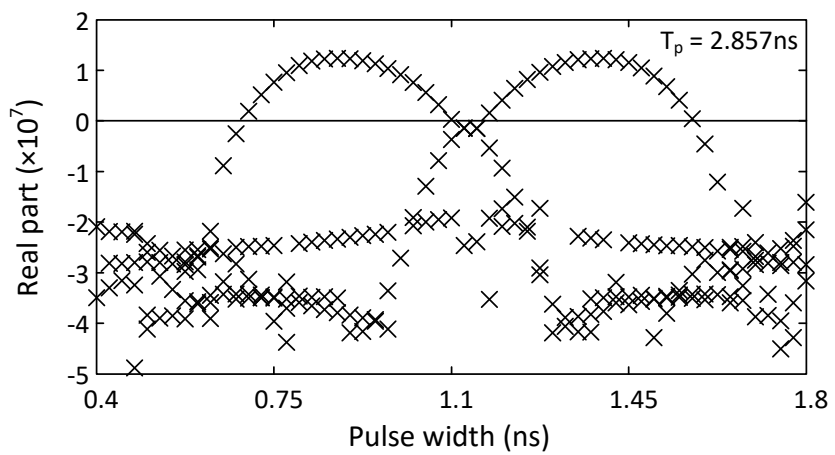

(b)

Fig. 9 Validation of the bifurcation loci in Fig. 8 with pole-zero identification. (a) For the pulse period $T_{p}=2.0 \mathrm{~ns}$. (b) For the pulse period $T_{p}=2.857 \mathrm{~ns}$.

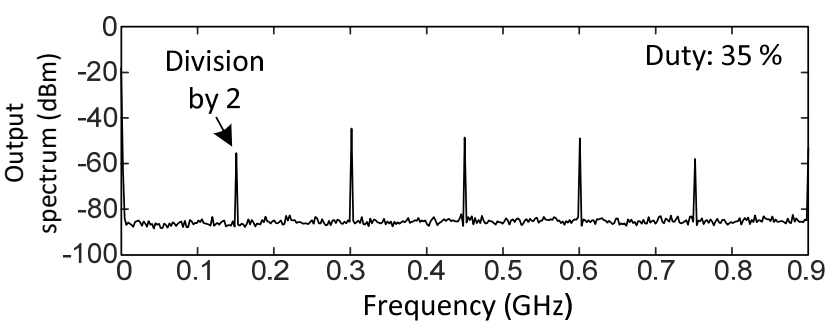

(a)

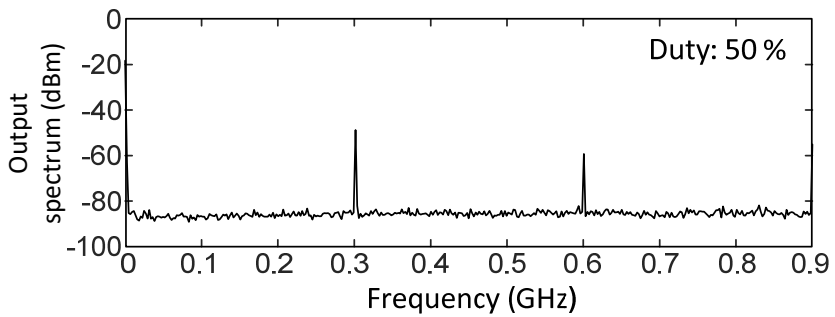

(b)

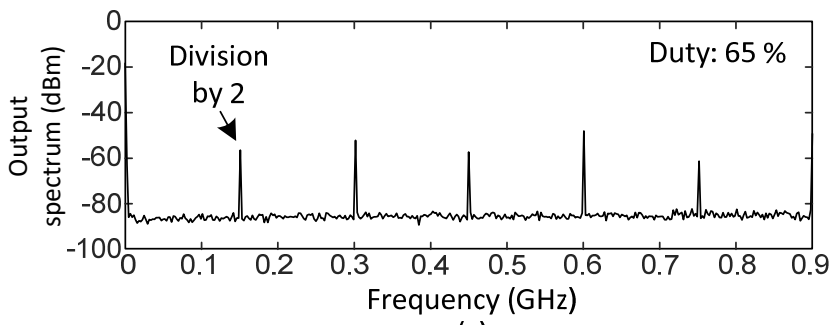

(c)

Fig. 10 Experimental spectra. (a) For $T_{p}=3.33$ ns and $w_{p}=1.167 \mathrm{~ns}$, exhibiting a frequency division by 2. (b) For $T_{p}=3.33 \mathrm{~ns}$ and $w_{p}=1.67 \mathrm{~ns}$, with stable behavior at the input pulse frequency. (c) For $T_{p}=3.33 \mathrm{~ns}$ and $w_{p}=2.167 \mathrm{~ns}$, exhibiting a frequency division by 2 .

\section{STABILITY ANALYSIS OF TRIBAND CLASS-S PA}

Based on the stability algorithms derived in Secs. II - IV, a more complex, multiband digital class-S power amplifier circuit is analyzed now. Fig. 11 presents the schematic of the PA which was presented in [42]. It includes a 4-stage GaNbased digital PA MMIC to amplify the binary input bit stream and a lumped-element tri-band filter at the output which reconstructs the wanted signals at the bands of $0.8,1.8$ and 2.6 $\mathrm{GHz}$. The presence of a multiband output filter is even more challenging for the stability (analysis) of the circuit as zero points in the output impedance characteristic may lead to several oscillations. The final-stage is realized in push-pull configuration where the two transistors $\mathrm{T}_{1 / 2}$ [Fig. 11(b)] switch the output voltage on and off between final-stage drain supply voltage $\left(+V_{D D}\right)$ and ideally $0 \mathrm{~V}$ (voltage-mode).

The stability of this class-S PA has been analyzed exhaustively in the course of this work. All simulations and measurements have been carried out with a pulse-width modulated signal (PWM). As PWM reaches maximum coding efficiency the results give a good estimation of the PA potential. Initially a small-signal stability analysis was carried out (in the absence of the input source), under a full variation of the bias sources, covering the excursions by the state variables when using the driving source. Different transfer 
functions have been tested to avoid a wrong stability conclusion due to possible pole-zero cancellations [23].

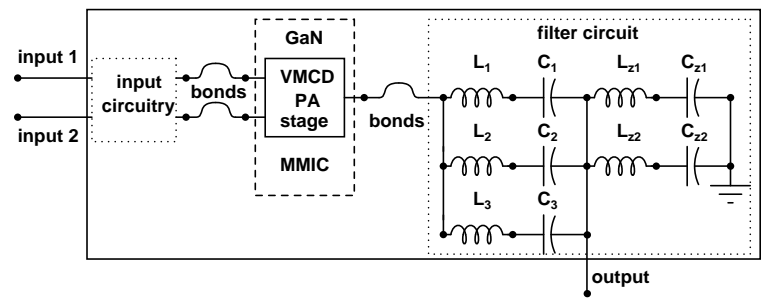

(a)

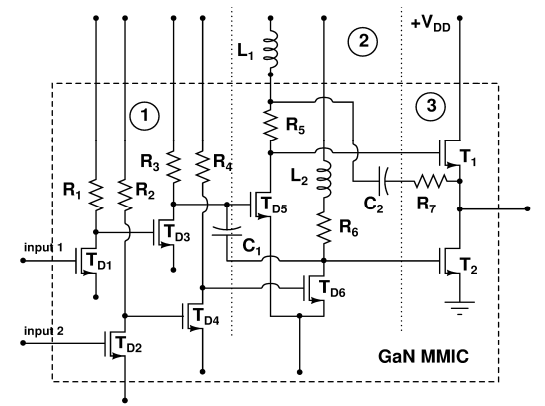

(b)

Fig. 11. (a) Tri-band class-S PA including lumped-element tri-band filter and (b) schematic of 4-stage GaN digital power amplifier stage (VMCD PA stage in 11 (a)).

The current source was alternatively connected to the gate and drain terminals of all the transistors. In all cases, the same stability predictions (stable, unstable and frequencies of the dominant poles) were obtained. The amplifier turned out to be stable for all bias values. Next it was analyzed for the nominal biasing conditions, using the Fourier Transform of a bit sequence "01" (simple PWM signal) as input signal, with amplitude $V_{\text {in }}=0.5$ volts.

Initially, a comparison of the frequency and time-domain results was performed using a simplified version of the amplifier, since it was not possible to analyze the stability of the full triband amplifier in time domain. The comparison with the simplified version showed an excellent agreement. The $\mathrm{HB}$ analysis has been carried out with 15 and 31 harmonics to enable an optimum performance of the Krylov solver, obtaining similar results in both cases. Fig. 12 presents the variation of the real part of the dominant poles versus the input bitrate. For good identification accuracy, the whole bitrate range was split into 5 sub-intervals, merging the results, which evidences a continuous variation of the dominant poles. The analysis was repeated for other input amplitudes, bias conditions and most significant elements values, and the amplifier remained stable in all cases. This stable behavior is attributed to very small feedback effects coming from the parasitic elements of the transistor devices.

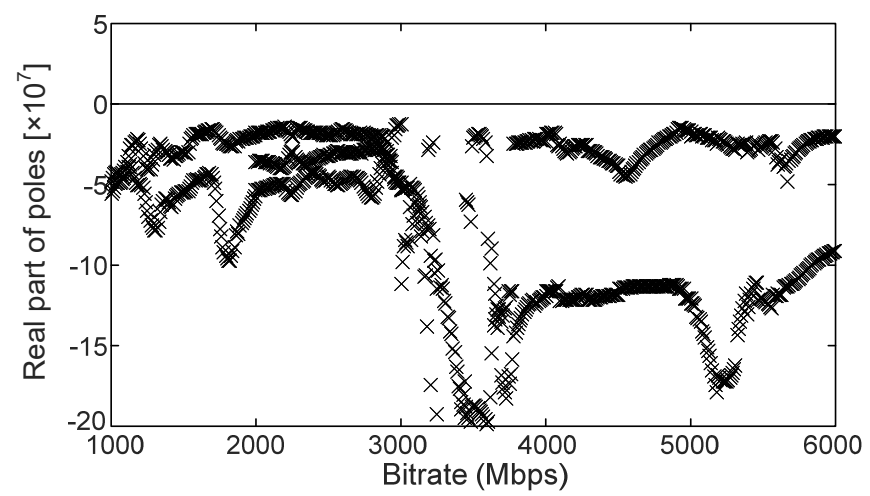

Fig. 12. Large-signal stability analysis considering a bit sequence " 01 ", with amplitude $V_{\text {in }}=0.5$ volts, versus variations in the input bitrate. The continuity of the poles should be noted.

A more detailed analysis has been carried out for two input signals of rectangular waveform: at $f_{S}=800 \mathrm{MHz}$ (i.e., 1.6 Gbps) and at $f_{S}=1.85 \mathrm{GHz}(3.7 \mathrm{Gbps})$. Fig. 13 compares the pole loci obtained for these two frequencies [in (b) and (c)] with the locus obtained when suppressing the input signal [in (a)], through a stability analysis of the dc solution. In dc there are dominant poles at $f_{o 1}=179.8 \mathrm{MHz}, f_{o 2}=435.4 \mathrm{MHz}$, and $f_{o 3}=1.384 \mathrm{GHz}$. At $800 \mathrm{MHz}$, the dominant poles (comprised between 0 and $f_{\text {in }}$ ) have the frequency values $90 \mathrm{MHz}$ and 180 $\mathrm{MHz}$. At $1.85 \mathrm{GHz}$, the poles are more distant from the imaginary axis and their frequencies are $100 \mathrm{MHz}$ and 280 $\mathrm{MHz}$. The stability has also been analyzed versus the duty cycle, obtaining stable behavior in all cases. Simulations for $f_{\mathrm{S}}$ $=800 \mathrm{MHz}$ are shown in Fig. 14 .

To prove the stability of the DPA it was measured exemplary with full-scale ("10") PWM input signals of $f_{\mathrm{S}}=$ $800 \mathrm{MHz}$ and $f_{S}=1.85 \mathrm{GHz}$. Fig. 15 shows the measured spectra. One clearly observes stable behavior as both output spectra $\left(f_{1}, f_{2}\right)$ show typical PWM spectrum with its typical harmonic distribution. No oscillation is identified. 


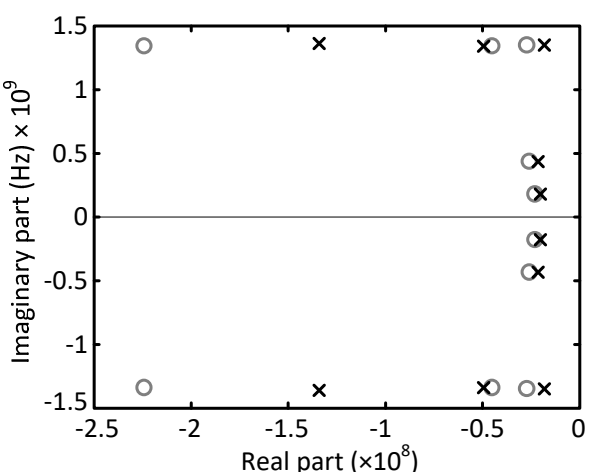

(a)

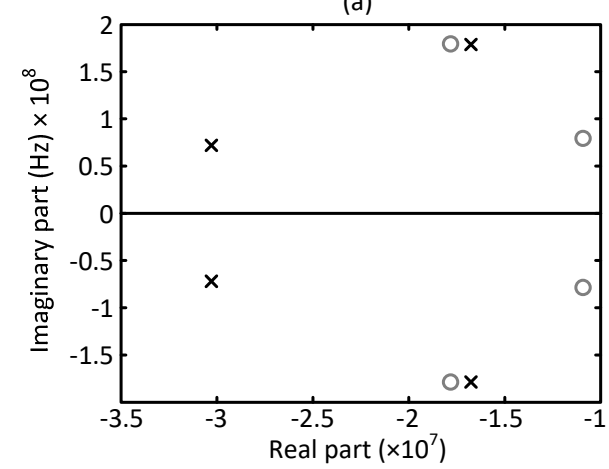

(b)

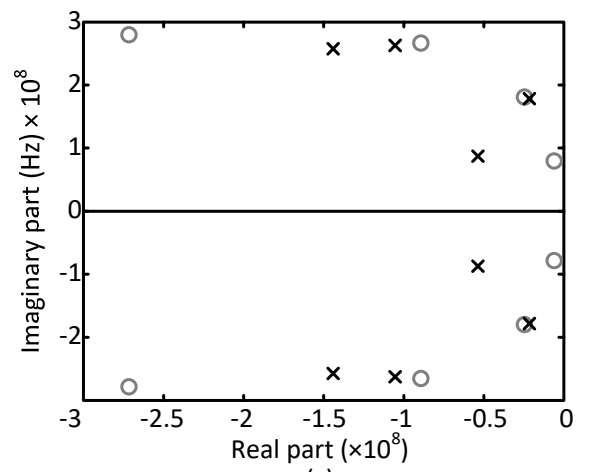

(c)

Fig. 13 Circuit dominant poles. (a) dc solution. (b) Input signal at 800 $\mathrm{MHz}$. (c) Input signal at $1.85 \mathrm{GHz}$.

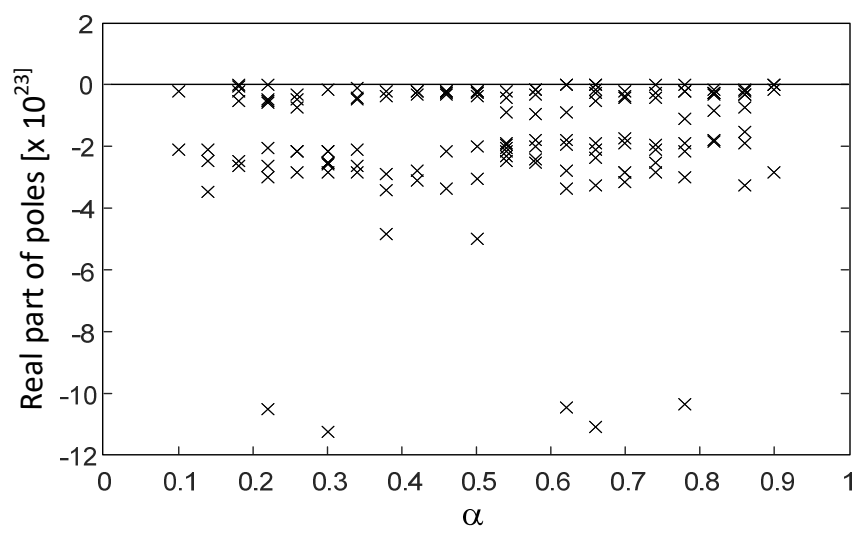

Fig. 14 Stability analysis of the PA in Fig. 11 versus the parameter $\alpha$ for $f_{\mathrm{S}}=800 \mathrm{MHz}$.

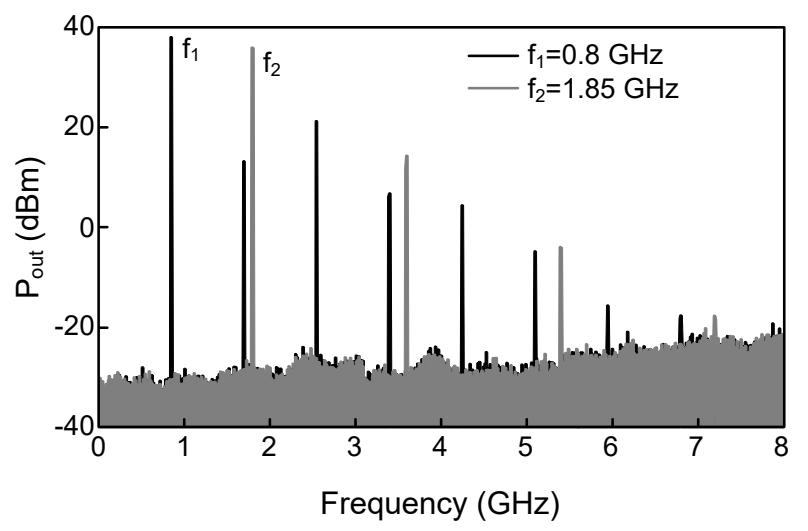

Fig. 15. Measured output spectra of triband DPA for encoded signal frequencies of $0.8 \mathrm{GHz}$ (black, $f_{1}$ ) and $1.85 \mathrm{GHz}\left(\mathrm{red}, f_{2}\right.$ ).

\section{CONCLUSION}

A stability analysis of digital microwave power amplifiers of class-D and class-S type, driven with binary pulse trains, has been presented for the first time. Frequency-domain techniques have been applied to investigate the impact of the input signal on the circuit natural frequencies. As has been shown, the average value of the input pulse has a relevant impact on the stability properties, since it leads to a change in the critical poles, due to its biasing effects. In class-D topologies, the instability may originate in either one or another of the two amplifier branches, with the other one responding to the input pulse, as well as the oscillation signal. Bifurcation detection techniques have been successfully applied for an efficient detection of the unstable intervals in terms of the various pulse parameters. Two different types of loci have been obtained, and the impact of the circuit topology and odd-mode excitation in the shape and symmetries of the loci has been studied. The circuit has been manufactured and measured obtaining very good agreement with the simulation results. The stability investigation has also been applied to a triband $(0.8,1.8$ and $2.6 \mathrm{GHz})$ class-S power amplifier, analyzing the stability properties under variations of the bitrate. A stable behavior has been obtained, which agrees with the experimental findings.

\section{ACKNOWLEDGEMENT}

Authors are grateful to Mr. Ignacio Ruiz from Keysight Technologies.

\section{REFERENCES}

[1] Ericsson (2015), Ericsson Mobility Report, [Online]. Available: https://www.ericsson.com/res/docs/2015/mobilityreport/ericsson-mobility-report-nov-2015.pdf

[2] W.-Y. Kim et al., "An Efficient Voltage-Mode Class-D Power Amplifier for Digital Transmitters with Delta-Sigma Modulation," in IEEE MTT-S Int. Microwave Symp. Dig., Baltimore, MD, 2011, THPH-1.

[3] H. Nakamizo et al., "Over 65\% PAE GaN Voltage-Mode Class D Power Amplifier for $465 \mathrm{MHz}$ Operation Using Bootstrap 
Drive," IEEE Topical Conference on Power Amplifiers for Wireless and Radio Applications Conference Publications, 2015.

[4] R. Ma, "A Review of Recent Development on Digital Transmitters with Integrated GaN Switch-Mode Amplifiers," in IEEE Int. Symp. on RFIT, Sendai, Japan, 2015, pp. 73-75.

[5] A. Wentzel et al., "A Flexible GaN MMIC Enabling Digital Power Amplifiers for the Future Wireless Infrastructure," in IEEE MTT-S Int. Microwave Symp. Dig., Phoenix, AZ, 2015, TH2B-5.

[6] A. Wentzel et al., "A Dual-Band Voltage-Mode Class-D PA for $0.8 / 1.8 \mathrm{GHz}$ Applications," in IEEE MTT-S Int. Microwave Symp. Dig., Seattle, WA, 2013, TH3A-3.

[7] Y. Zhang et al., "The Topology Analysing of a Cascade Multilevel Class-D Power Amplifier," in International Conference on Control Engineering and Communication Technology, Liaoning, 2012, pp. 109-112.

[8] Y. Choi et al., "A 0.018\% THD+N, 88-dB PSRR PWM Class-D Amplifier for Direct Battery Hookup," in IEEE J. Solid-State Circuits, vol. 47, no. 2, pp. 454-463, Feb. 2012.

[9] D. Cartasegna et al., "A design methodology for high-order classD audio amplifiers," Analog Integrated Circuits and Signal Processing, vol. 78, no. 3, pp 785-798. Mar. 2014.

[10] S. M. Cox et al., "A second-order class-D audio amplifier," SIAM Journal on Applied Mathematics, 71 (1). pp. 270-287. Jan. 2005.

[11] J. Jugo, J. Portilla, A. Anakabe, A. Suárez, J. M. Collantes, "Closed-loop stability analysis of microwave amplifiers," IEEE Electronics Letters, vol. 37, no. 4, pp. 226-228, Feb. 2001.

[12] N. Ayllon et al., "Systematic approach to the stabilization of multitransistor circuits", IEEE Trans. Microw. Theory Tech., vol. 59, no. 8, pp. 2073-2082, Aug. 2011.

[13] V. Rizzoli and A. Neri, "State of the art and present trends in nonlinear microwave CAD techniques," IEEE Trans. Microw. Theory Tech., vol. 36, no. 2, pp. 343-356, Feb., 1988.

[14] R. Quéré, E. Ngoya, M. Camiade, A. Suárez, M. Hessane J. Obregón, "Large signal design of broadband monolithic microwave frequency dividers and phase-locked oscillators," IEEE Trans. Microw. Theory Tech., vol. 41, no. 11, pp. 19281938, Nov. 1993.

[15] J. C. Sarkissian, M. Camiade, P. Savary, A. Suárez, R. Quéré and J. Obregón, "A 60-Ghz HEMT MMIC analog frequency divider by two," IEEE Journal of Solid State Devices, vol. 30, no.10, Oct.1995, pp. 1062-1067.

[16] A. Suárez, S. Jeon and D. Rutledge, "Stability analysis and stabilization of power amplifiers," IEEE Microwave Magazine, vol. 7, pp. 51-65, Oct., 2006.

[17] A. Suárez, Analysis and Design of Autonomous Microwave Circuits, IEEE-Wiley, Hoboken, (NJ) Jan. 2009.

[18] R. W. Freund, "Passive reduced-order modelling via Krylovsubspace methods," in IEEE International symposium on Computer-Aided Control System Design, Anchorage, AK, 2000, pp. 261-266.

[19] R. Melville, H. G. Brachtendorf, "An effective procedure for multi-tone steady-state analysis of mixers," in 8th IEEE Conference on Electronics Circuits and Systems, Malta, 2001, vol. 3, pp. 1449-1453.

[20] L. Chua, A. Ushida, "A switching parameter algorithm for finding multitple solutions of nonlinear resistive circuits," International Journal of Circuit Theory and Applications 4(3), pp. 215-239, Jul. 1976.

[21] T.S. Parker, L.O. Chua, Practical Numerical Algorithms for Chaotic Systems, Springer-Verlag, New York, 1989.

[22] Y. Kuznetsov, Elements of applied bifurcation theory, Springer (third edition), 2005.

[23] R. Courant, F. John, Introduction to Calculus and Analysis, Volume II., Springer-Verlag, 1989.
[24] W. C. Rheinboldt, J. Burkardt, “Algorithm 596: A Program for a Locally Parameterized Continuation Process", ACM Trans Math. Software, 1983 9(2):236-241.

[25] L.T. Watson et al., "Algorithm 652 Hompack: A Suite of Codes for Glogally Convergent Homotopy Methods", ACM, Trans. Math. Software, vol. 13, no. 3, pp. 281-310, Sept. 1987.

[26] R.C. Melville et al., "Artificial Parameter Homotopy Methods for the DC Operating Point Problem", IEEE Trans. Comp-Aided Des. Integr. Circuits Syst., vol. 12, no. 6, Jun. 1993.

[27] A. Suárez, J. Morales, R. Quéré, "Synchronization analysis of autonomous microwave circuits using new global-stability analysis tools," IEEE Trans. Microw Theory Tech., vol. 46, no. 5, pp. 494-504, May 1998.

[28] A. Suarez, J. M. Collantes, "A new technique for chaos prediction in RF circuit design using harmonic-balance commercial simulators," IEEE Trans. Circuits Syst. I, Fundam. Theory Appl., vol. 46, no. 11, pp. 1413-1415, Nov. 1999.

[29] J. Guckenheimer and P. Holmes, Nonlinear Oscillations, Dynamical Systems and Bifurcations of Vector Fields. New York: Springer-Verlag, 1990.

[30] V. Rizzoli, A. Lipparini, "General stability analysis of periodic steady-state regimes in nonlinear microwave circuits," IEEE Trans. Microw. Theory Tech., vol. 33, no. 1, pp. 30 - 37, Jan. 1985.

[31] F. Ramírez, A. Suárez, I. Lizarraga, J.-M. Collantes, "Stability analysis of nonlinear circuits driven with modulated signals," IEEE Trans. Microw. Theory Tech., vol. 58, no. 4, pp. 929-940, Apr. 2010.

[32] E. Ngoya, R. Larcheveque, "Envelop transient analysis: A new method for the transient and steady state analysis of microwave communication circuits and systems," in IEEE MTT-S Int. Microwave Symp. Dig., San Francisco, CA, 1996, pp. 13651368.

[33] A. Gelb, W. Vander Velde, Multiple-input describing functions and nonlinear system design, McGraw-Hill, 1968.

[34] F. Bonani and M. Gilli, "Analysis of stability and bifurcations of limit cycles in Chua's circuit through the harmonic-balance approach," IEEE Trans. Circuits Syst. I, vol. 46, no. 8, pp. 881890, Aug. 1999.

[35] J. M. Collantes et al., "Stability verification of microwave circuits through Floquet multiplier analysis," in IEEE APCCAS, Taiwan, 2004, pp. 997-1000.

[36] G. Sarafian, B. Z. Kaplan, "The dynamics of parametric frequency divider and some of its practical implications," Nineteenth Convention of Electrical and Electronics Engineers, Israel, 1996, pp. 523-526, Nov. 1996.

[37] S. Basu and S. A. Maas, "Design and Performance of a Planar Star Mixer," IEEE Trans. Microw. Theory Tech., vol. MTT-41, no. 11, page 2028-30, Nov. 1993.

[38] J. M. Paillot et al., "A general program for steady state, stability, and FM noise analysis of microwave oscillators," in IEEE MTT-S Int. Microwave Symp. Dig., 1990, pp. 1287-1290.

[39] S. Wiggins, Introduction to Applied Nonlinear Dynamical Systems and Chaos, New York: Springer-Verlag, 1990.

[40] G. Ioos, D. Joseph, Elementary Stability and Bifurcation Theory, New York, Springer, 1980.

[41] J. M. T. Thompson, H. B. Stewart, Nonlinear dynamics and chaos, John Wiley \& sons, 2002.

[42] A. Wentzel et al., "A compact tri-band GaN voltage-mode class-D/S PA for future 0.8/1.8/2.6 GHz LTE picocell applications," in European Microwave Conference (EuMC) Dig., Paris, France, 2015, pp. 1160-1163. 


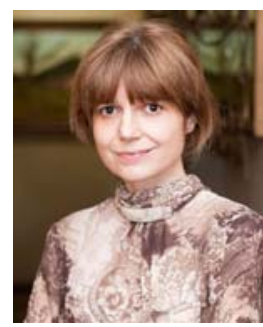

Almudena Suárez was born in Santander, Spain. She received the degree in electronic physics and the $\mathrm{Ph} . \mathrm{D}$. degree from the University of Cantabria, Santander, Spain, in 1987 and 1992, respectively, and the Ph.D. degree in electronics from the University of Limoges, France, in 1993. At present, she is a Full Professor at the University of Cantabria, and a member of its Communications Engineering Department. She has authored the book Analysis and design of autonomous microwave circuits for the publisher IEEE-Wiley and co-authored the book Stability analysis of microwave circuits for the publisher Artech-House. She belongs to the technical committees of the IEEE International Microwave Symposium and European Microwave Conference. She was an IEEE Distinguished Microwave Lecturer for the period 2006-2008. She is a member of the Board of Directors of EuMA and the Editor in Chief of International Journal of Microwave and Wireless Technologies from Cambridge University Press.

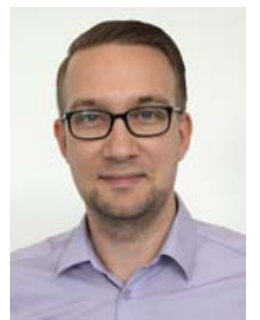

Andreas Wentzel (M'16) received the Dipl.-Ing. and the $\mathrm{Ph} . \mathrm{D}$. degree in electrical engineering from the Technical University of Berlin, Germany, in 2006 and 2011, respectively. He is presently the head of the digital PA lab of the Ferdinand-Braun-Institut (FBH) in Berlin, Germany. His research activities focus on the design of digital transmitter architectures including advanced switch-mode power amplifier concepts realized on $\mathrm{GaN}$, especially in class-S and class-D operation, as well as on optimized modulation schemes and filter structures suitable for this type of PAs.

Dr. Wentzel is member of the German IEEE MTT section as well as of the European Microwave Association (EuMA).



Wolfgang Heinrich received the diploma, $\mathrm{PhD}$, and habilitation degrees from the Technical University of Darmstadt, Germany. Since 1993, he has been with the Ferdinand-Braun-Institut (FBH) at Berlin, Germany, where he is head of the microwave department and deputy director of the institute. Since 2008, he is also professor at the Technical University of Berlin. His present research activities focus on MMIC design with emphasis on $\mathrm{GaN}$ power amplifiers, mm-wave integrated circuits, and electromagnetic simulation. Prof. Heinrich has authored or coauthored more than 300 publications and conference contributions. He has been serving the microwave community in various functions, e.g., as Distinguished Microwave Lecturer for the term 2003-2005, as General Chair of the European Microwave Week in Munich, 2007, and as Associate Editor of the IEEE Transactions on MTT from 2008 until 2010. Since 2010, he is President of the European Microwave Association (EuMA). 